EXTENDED REPORT

\title{
En face optical coherence tomography: a new method to analyse structural changes of the optic nerve head in rat glaucoma
}

\author{
L Guo, V Tsatourian, V Luong, A G Podolean, D A Jackson, F W Fitzke, M F Cordeiro
}

Br J Ophthalmol 2005;89:1210-1216. doi: 10.1136/bjo.2004.058941

See end of article for authors' affiliations

Correspondence to: Dr M F Cordeiro, Glaucoma and Óptic Nerve Head Research Group, Pathology, Institute of Ophthalmology, University College London, 11-43 Bath Street, London ECIV 9EL, UK; m.cordeiro@ucl.ac.uk

Accepted for publication 2 February 2005
Aim: To investigate en face optical coherence tomography (eOCT) and its use as an effective objective technique for assessing changes in the glaucomatous rat optic nerve head (ONH) in vivo, and compare it with confocal scanning laser ophthalmoscopy (cSLO).

Methods: 18 Dark Agouti (DA) rats with surgically induced ocular hypertension were imaged with eOCT and cSLO at regular intervals. Assessment included three dimensional (3D) topographic reconstructions, intensity z-profile plots, a new method of depth analysis to define a "multilayered" structure, and scleral canal measurements, in relation to the degree of intraocular pressure (IOP) exposure.

Results: The increased depth resolution of the eOCT compared to the CSLO was apparent in all methods of analysis, with better discrimination of tissue planes. This was validated histologically. eOCT demonstrated several significant changes in imaged rat $\mathrm{ONH}$ which correlated with IOP exposure, including the area of $\mathrm{ONH}(p<0.01)$, separation between retinal vessel and scleral layers $(p<0.05)$, and anterior scleral canal opening expansion $(p<0.05)$.

Conclusion: eOCT appears to be effective in assessing rat $\mathrm{ONH}$, allowing detailed structural analysis of the multilayered $\mathrm{ONH}$ structure. As far as the authors are aware, this is the first report of scleral canal expansion in a rat model. They suggest $\mathrm{eOCT}$ as a novel method for the detection of early changes in the $\mathrm{ONH}$ in glaucoma.
G laucoma is characterised by visual field loss, retinal ganglion cell death, and optic nerve head (ONH) damage, with elevated intraocular pressure (IOP) being defined as the most important treatable risk factor. ${ }^{12}$ A major advance in the field of glaucoma has been the development of imaging techniques that allow objective measurements of the ONH. Until recently, these techniques have not been able to be applied accurately to the increasingly popular rat model of glaucoma, because of the optics and the small size of rat eyes. In addition, three dimensional (3D) in vivo imaging and its correlation with morphometric and histological data has not previously been possible. ${ }^{3-7}$

Currently, the most popular instrument for imaging the $\mathrm{ONH}$ is the confocal scanning laser ophthalmoscope (cSLO), of which the Heidelberg retinal tomograph (HRT) is the best known, producing a colour coded surface topographical map. A recent development in high resolution fundal imaging has been the technique of en face optical coherence tomography (eOCT). ${ }^{8}$ Unlike conventional OCT, ${ }^{9}{ }^{10}$ eOCT processes a series of transverse scans through tissue at equally distributed and specific depths, determined by modern optical sources capable of achieving depth resolutions of up to $3 \mu \mathrm{m} .^{8}$

Although optic disc "cupping" has been previously demonstrated in the rat model of glaucoma, an effective objective imaging technique is yet to be established..$^{11}$ This is important as the rat model is increasingly recognised as a useful tool for studying glaucoma, with similarity to human disease. ${ }^{12}$ Recently, Burgoyne and co-workers have demonstrated histological changes in the scleral canal in early experimental glaucoma, ${ }^{13}{ }^{14}$ which has not been able to be assessed objectively before in vivo in the rat model, even though the HRT has been previously applied in the investigation of rat $\mathrm{ONH}$ changes. ${ }^{11}$

Because of the high depth resolution of the eOCT, we have investigated its application in experimental glaucoma and assessed scleral canal changes in this study, comparing it with the more commonly used imaging device-the scanning laser ophthalmoscope. The aim of this study was to investigate the new technique of eOCT in the analysis of glaucomatous rat $\mathrm{ONH}$ and compare it to the cSLO. Several different methods were used in the comparison, including a newly devised approach of depth analysis that enabled assessment of the "multilayered" anatomical structure of the rat $\mathrm{ONH}$.

\section{METHODS}

\section{In vivo study design}

Adult male Dark Agouti (DA) rats weighing 150-200 g were treated with procedures approved by local ethics committee and the UK Home Office.

Eighteen animals underwent uniocular hypertension surgery, as described by Morrison. ${ }^{15}$ Briefly, under general anaesthesia, all animals had the IOP in the left eye elevated by injection of $50 \mu \mathrm{l}$ of hypertonic saline solution (1.80 M) into the episcleral veins with a polypropylene ring around the equator of the globe to prevent hypertonic saline draining and damaging surrounding tissues. ${ }^{15}$ The animals were kept in constant low light and the IOP was taken at the same time of day on all test days. The IOP of both eyes in each rat was measured under inhalational anaesthesia of a mixture of oxygen and isofluorane, using a Tonopen XL preoperatively and at regular weekly intervals postoperatively. IOP elevation in the eye which had undergone surgery was calculated from

Abbreviations: ASCO, anterior scleral canal opening; CSLO, confocal scanning laser ophthalmoscopy; 3D, three dimensional; DA, Dark Agouti; $\mathrm{eOCT}$, en face optical coherence tomography; FWHM, full width at the half maximum; HRT, Heidelberg retinal tomograph; IOP, intraocular pressure; LC, lamina cribrosa; ODA, optic disc area; $\mathrm{ONH}$, optic nerve head; PSCO, posterior scleral canal opening 

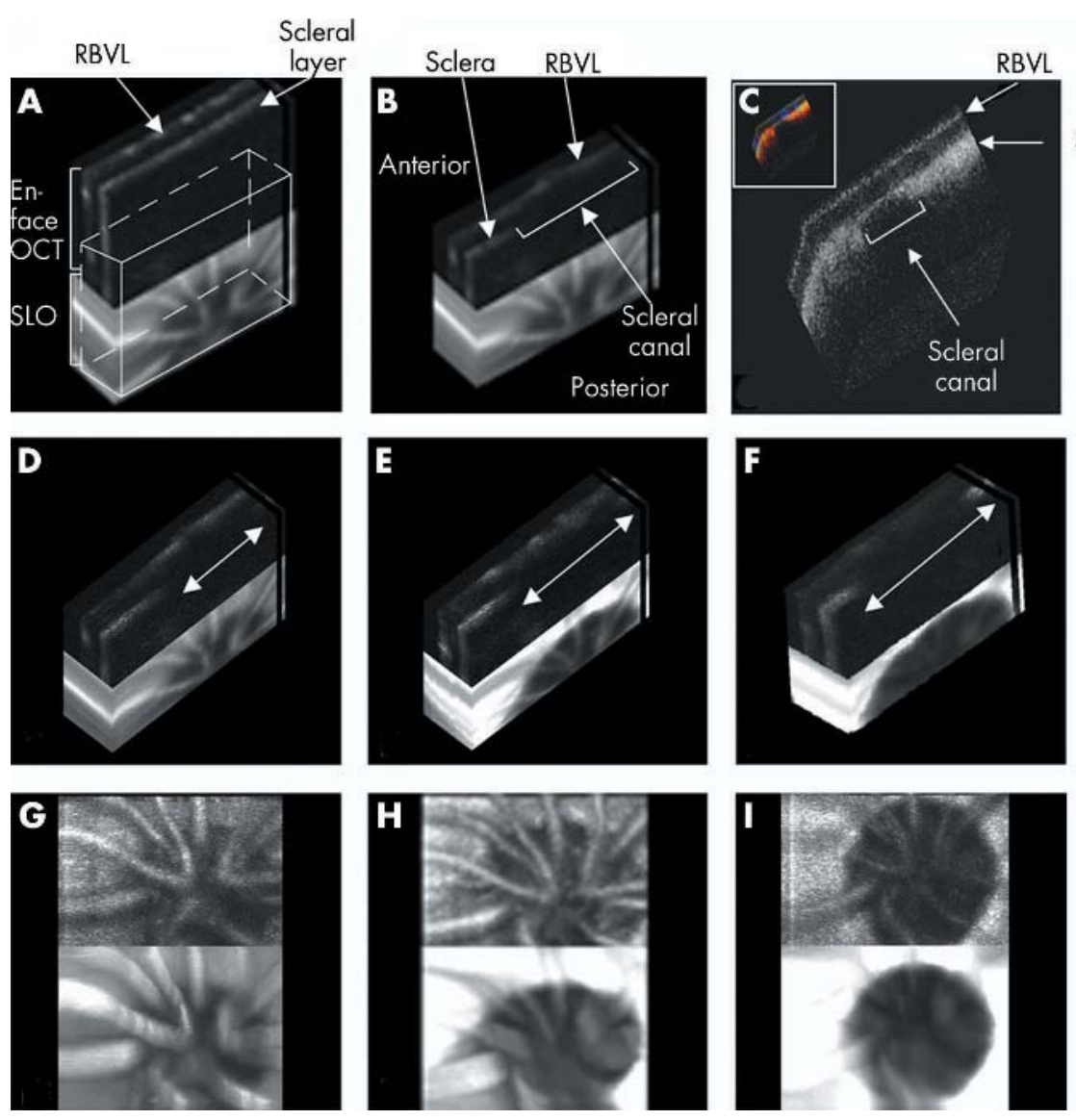

Figure 1 Image analysis method 1. En face OCT (eOCT) imaging of optic nerve head $(\mathrm{ONH})$ in glaucomatous rats with $3 \mathrm{D}$ reconstructive blocks of a Sclera series of eOCT sections. The images consist of an eOCT image above and a confocal scanning laser

ophthalmoscopy (cSLO) image below. Retinal blood vessel layers (RBVL) and scleral layer are easily demonstrated as indicated on the EOCT 3D

reconstructive image (A). The edges of the $\mathrm{ONH}$ are clearly visible in the cross section through the $\mathrm{ONH}$ taken from $\mathrm{A}$ (B), and validated by confocal 3D reconstruction of immunohistochemistry ( $C$, colour inset). Progressive widening of the scleral canal was seen in all glaucomatous rats (arrows in D-F). Typical examples of eOCT images (3D reconstructions D-F, XY projections $G-$ I) are shown in the same glaucomatous animal at baseline $(D, G), 2$ months $(E$, $H$ ) and 3 months $(F, I)$ with IOP integral of $0,542.65$, and $665.85 \mathrm{~mm} \mathrm{Hg}$ days, respectively. the difference between the IOP in the operated and control eyes at each time point and the $\Delta \mathrm{IOP}$ integral was then calculated for each animal. ${ }^{16} 17$

\section{Imaging of $\mathrm{ONH}$}

Imaging of the $\mathrm{ONH}$ was performed under general anaesthetic at baseline and at regular intervals postoperatively, as we have previously described. ${ }^{16}{ }^{17}$ Briefly, rats with dilated pupils were placed in a stereotaxic frame and the $\mathrm{ONH}$ centred in the image frame. To achieve longitudinal analysis of changes with time, each rat had both eyes assessed repeatedly by the eOCT and CSLO at the same time point. A total of $150 \mathrm{C}$-scan eOCT images were acquired at $5 \mu \mathrm{m}$ intervals covering $0.75 \mathrm{~mm}$ in depth using a superluminescent diode as the light source (Applied Optics Group Kent University, UK). ${ }^{8}$ A simultaneously produced confocal SLO image permits the centring of the ONH and monitoring of eye movements. A total of 32 transversal images at $60 \mu \mathrm{m}$ intervals were then acquired using a Zeiss prototype laser scanning ophthalmoscope (Carl Zeiss, Oberkochen, Germany). ${ }^{18}$

\section{Image analysis method 1 (fig 1)}

Pairs of eOCT and cSLO digitised images were collected from each animal at the same time point, and analysed using 3D Doctor software (Able Software Corp. Lexington, MA, USA). The optic disc area (ODA) was determined from 3D reflectance image reconstructions by calculating the area enclosed by a manually drawn contour line outlining the clearly demarcated edges of the optic disc (fig lG-I). The change in ODA was calculated from the difference between recorded and baseline readings.
Image analysis method 2 (figs 2 and 3)

3D eOCT and CSLO reconstructions were also analysed using in-house designed software, as shown in figure 2 . Conventional surface topography represents a single peak, usually symmetrical confocal z-profile, with the position of the profile maximum defining the location of the light reflecting surface along the $\mathrm{z}$-axis. It relies on the calculation of the centre of gravity of the zprofile and deals with asymmetric profiles by using a proprietary algorithm. Since eOCT clearly discriminates several layers, it produces a multi-peak z-profile, which makes analysis with conventional surface topography inapplicable.

Therefore, we developed a new method constructing a histogram where the total number of $(x, y)$ locations with peak intensity at a specific depth (vertical axis) are represented as a function of depth (horizontal axis). Hence, it defines the total number of pixel locations with the same depth of reflecting surface at any specific depth. As the number of locations (total pixels per image) is different for the SLO (74 800 pixels) and eOCT (153 600 pixels), the total number of $(x, y)$ locations with peak intensity was divided by the maximum number of locations at peak intensity, to create a scale between 0 and 1 (normalised number of pixels, figs $3 \mathrm{~A}, \mathrm{~B}$ ), in order to allow for direct comparisons between differing imaging techniques and different eyes. All histograms were then centred at the minimum between two peaks. The Gaussian approximation was used to find the full width at the half maximum (FWHM) of each peak (fig 3C). In addition, the separation between peaks was calculated as the distance between maximums of each Gaussian curve (fig 3C). 
eOCT

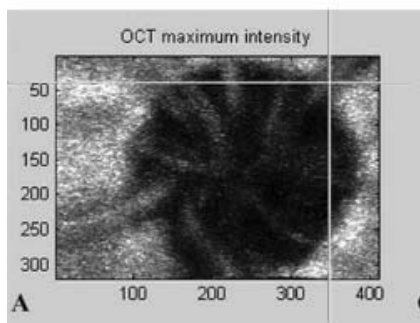

G
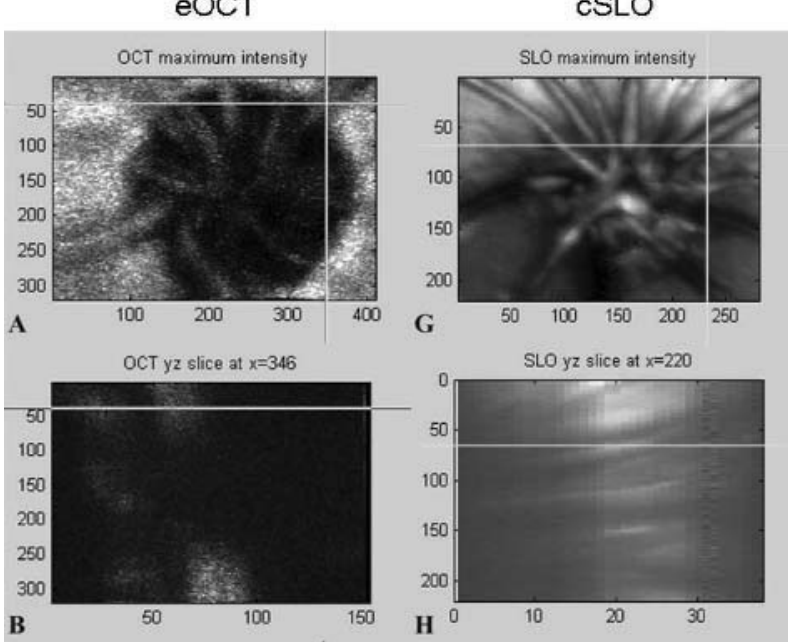

OCT $x z$ slice at $y=37$
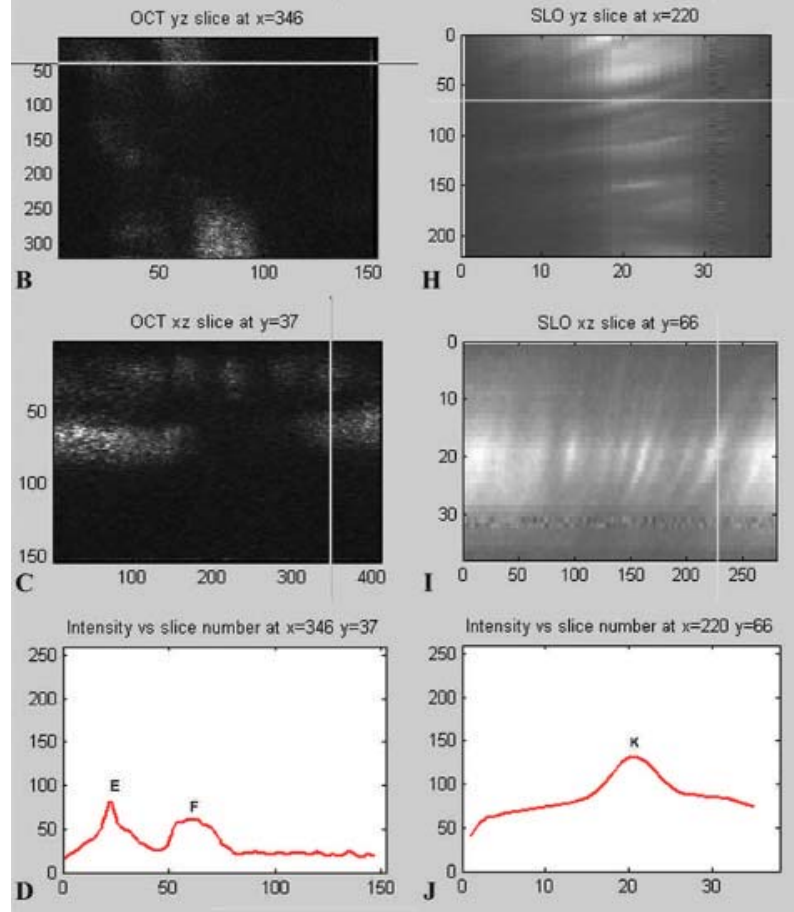

SLO $x z$ slice at $y=66$
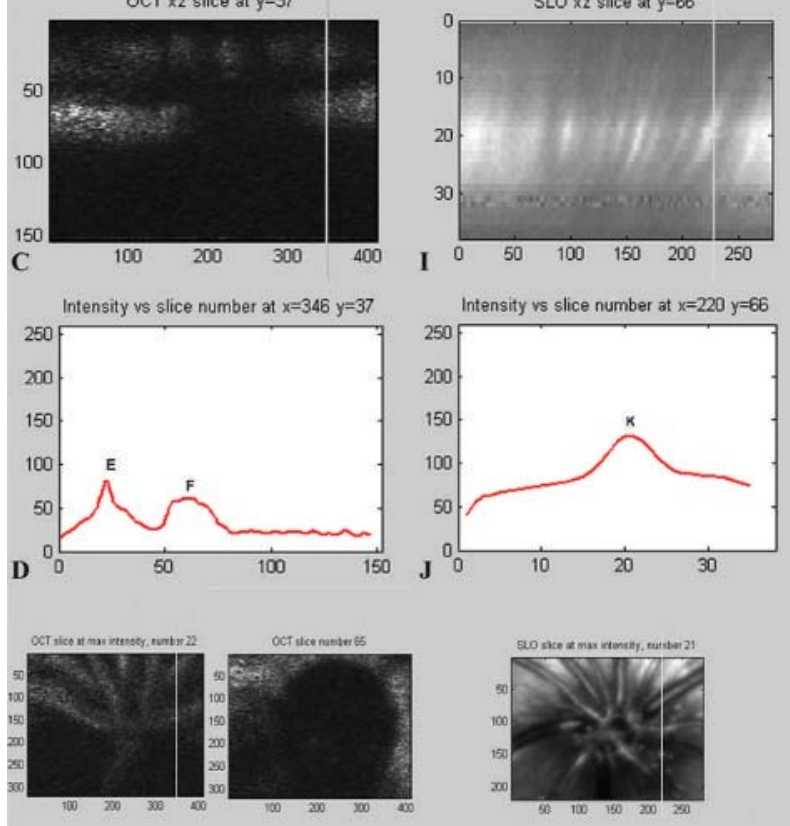

F

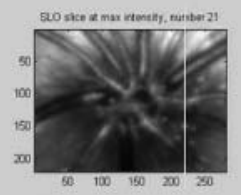

K
Figure 2 Image analysis method 2 of eOCT (A-F) and cSLO (G-K) images. Reflectance maps (akin to conventional surface topography) show the same eye of the same animal analysed with eOCT (A) and cSLO (G). Corresponding points, selected as shown by the cross intersection in $A$ and $G$, are further analysed using longitudinal slice reconstructions in the $y-z$ axis $(B, H)$ and $x-z$ axis $(C, I)$. The corresponding confocal $z$-profile plots are shown in (D) and $(J)$. A double peak z-profile was obtained with $\mathrm{eOCT}$ (D) but only a single peak $\mathrm{K}$ obtained with cSLO (J). Transversal optical slices corresponding to the peaks, as indicated in (D) and $(J)$, are shown with eOCT (E, F) and cSLO (K).

Image analysis method 3 (fig 4)

This method used the longitudinal slice images produced in method 2 (see fig $2 \mathrm{C}$ ), and consequently was only appropriate to eOCT. Longitudinal slices were taken through the middle of the optic disc, as determined from the reflectivity maps of each eye. The anterior scleral canal opening (ASCO) was defined as the maximal opening diameter in the anterior surface of the sclera, as delineated in figure 4A. Likewise, the posterior scleral canal opening (PSCO) was defined as the maximal opening diameter in the posterior surface of the sclera. For an estimate of the effect of compression on the scleral canal, we measured the thickness of the edges
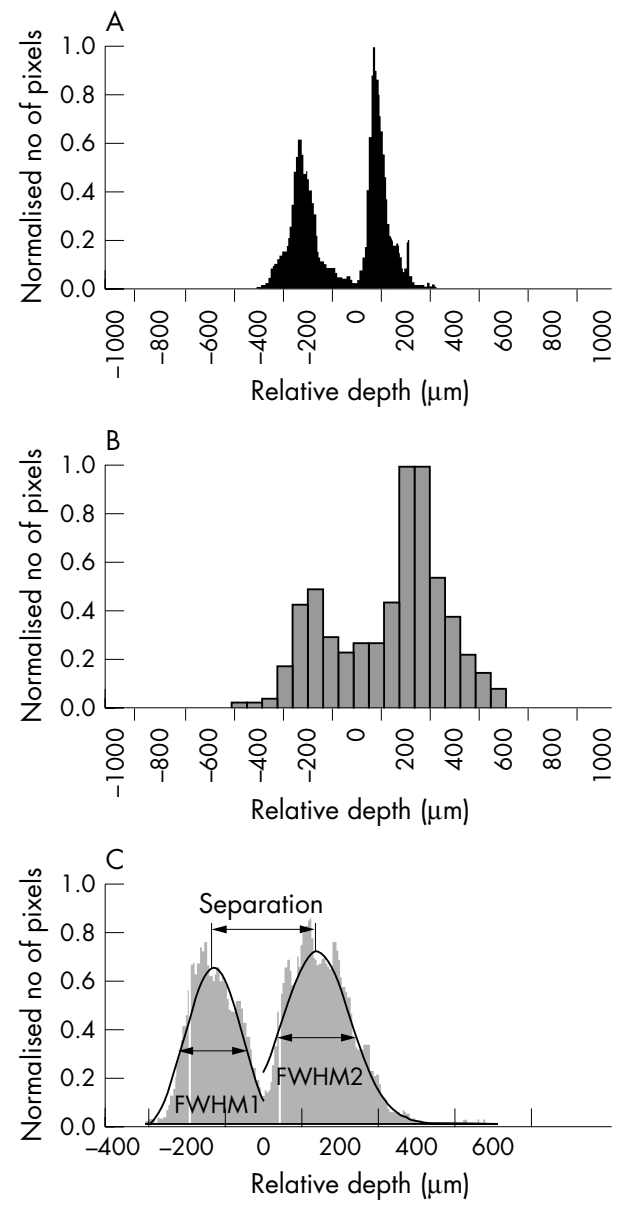

Figure 3 Image analysis method 2. Multisurface depth analysis generating histograms for $\mathrm{EOCT}(\mathrm{A})$ and $\mathrm{CSLO}(\mathrm{B})$ of the same eye in the same animal. Histograms represent the number of $(x, y)$ locations of the same depth of the reflecting surface (vertical axis) as a function of its depth (horizontal axis). All histograms are centred at the minimum between two peaks and normalised to the highest peak. Compared to cSLO, eOCT produced sharper and narrower peaks. For the analysis, we only included eyes with a double peak histogram for both eOCT and cSLO, where each peak could be approximated by a Gaussian curve. A typical Gaussian curve fit is shown in (C), from which the full width at the half maximum of the first (FWHM1) and second (FWHM2) peaks were calculated, as well as the distance between the two peaks (separation).

(fig 4A, " $\mathrm{t}$ ") of the scleral canal. Figure 4B shows these corresponding measurements in the histological section through the $\mathrm{ONH}$ of a normal eye.

\section{Histology}

Animals were sacrificed immediately following in vivo imaging at 3 months of study. Eyes were enucleated, fixed, and dissected ${ }^{16}{ }^{17}$ and $2 \mathrm{~mm}$ diameter full thickness specimens centred around the ONH obtained. The retinal nerve fibre layer was identified using mouse anti-rat neurofilament $200 \mathrm{kDa}$ antibody (NF-200, Sigma, UK, 1:1000). ${ }^{19}$ Scleral and nuclei labelling was performed with cirius red (PSR, Sigma) and 4',6'-diamidino-2-phenylindole (DAPI, Sigma, 1:2500). $3 \mathrm{D}$ reconstructions were obtained from images acquired using confocal microscopy. ${ }^{16}{ }^{17}$

\section{Statistical analysis}

All methods were assessed for reproducibility and accuracy, using the coefficient of variation to assess interobserver and intraobserver variation, with multiple observers (LG, AM, MFC, VT). ${ }^{20}$ 

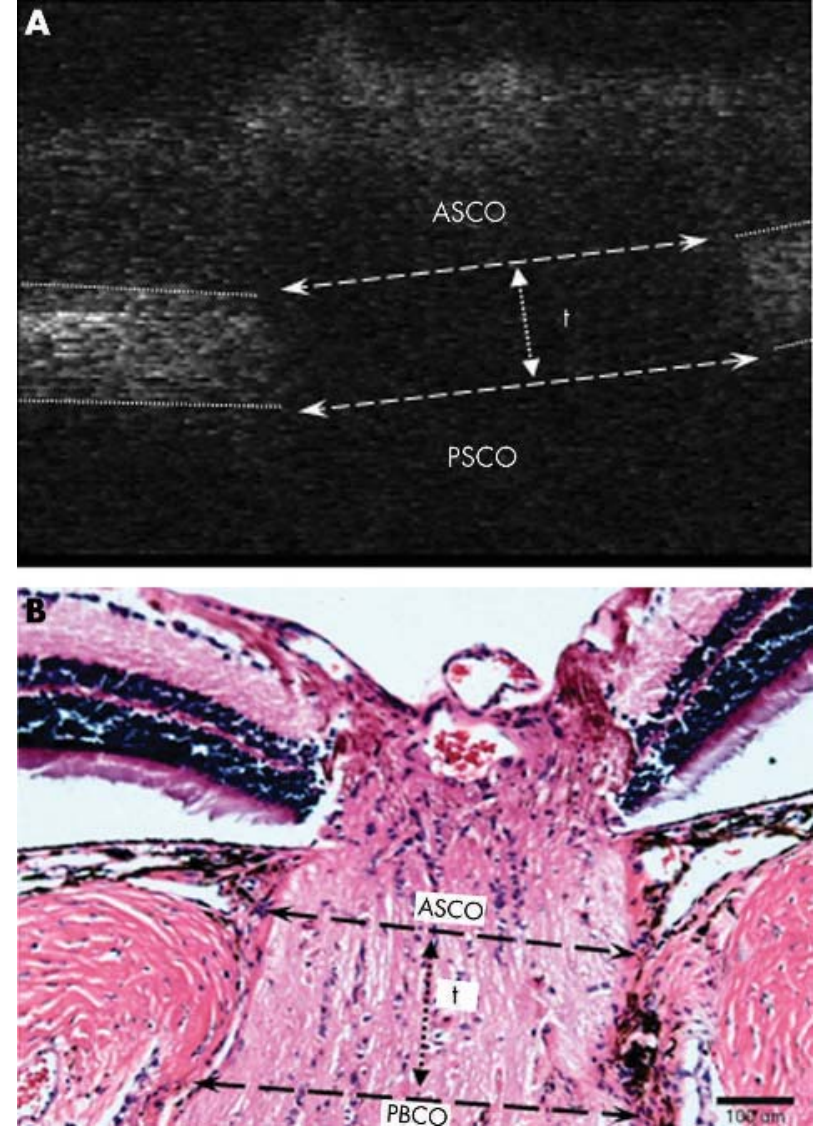

Figure 4 Image analysis method 3. A typical longitudinal slice from a $3 \mathrm{D} \mathrm{eOCT}$ image in which the anterior scleral canal opening (ASCO), posterior scleral canal opening (PSCO) and scleral canal thickness $\left("{ }^{\prime \prime}\right)$ are marked as indicated (A). Corresponding measurements in the

paraffin cross section through the ONH of a normal rat eye stained with haematoxylin and eosin, are shown for comparison (B).

Statistical analysis was performed to assess correlation of indicated parameters in the above three methods in relation to integral $\triangle \mathrm{IOP}$, using Pearson's correlation coefficient. ${ }^{17}$ ANOVA was used to investigate differences between eOCT and cSLO techniques.

\section{RESULTS}

Method 1: change in optic disc area

3D reconstructions demonstrate the high depth resolution of eOCT permitting the identification of distinct retinal and scleral layers (fig 1A, 1B). The edges of the $\mathrm{ONH}$ (scleral canal) are clearly visible in the cross section block through the ONH (fig lB). These same structures identified in eOCT were seen in corresponding histological 3D blocks (fig IC). Comparison between thickness of the posterior sclera with $3 \mathrm{D}$ reconstructions from eOCT $(88.54(\mathrm{SD} 2.59) \mu \mathrm{m})$ and confocal microscopy $(77.29(6.23) \mu \mathrm{m})$, suggest some tissue shrinkage with fixation processing. Progressive widening of the scleral canal was seen in all glaucomatous eyes, with typical examples shown in figures $1 \mathrm{D}-\mathrm{I}$.

Interobserver and intraobserver coefficients of variation were similar to those previously published for $\mathrm{HRT},{ }^{20}$ being 4.4 and 2.6 respectively.

Comparison between eOCT and cSLO in terms of change in ODA, showed a much narrower range for eOCT. Although there was no significant difference between the methods (fig 5C, ANOVA $p=0.2180$ ), we found no correlation either (fig 5C, Pearson's correlation coefficient).
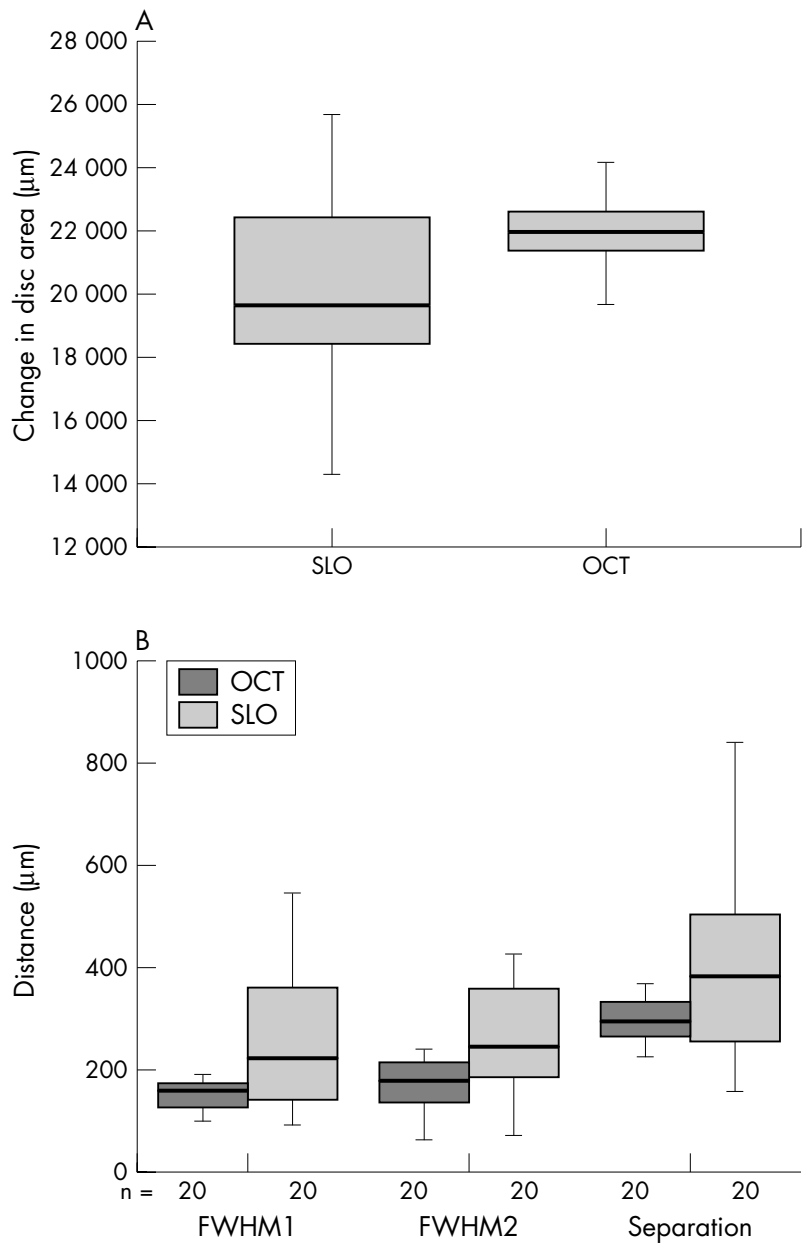

\begin{tabular}{|c|c|c|c|c|c|}
\hline \multicolumn{2}{|l|}{ C } & FWHMI & FWHM2 & Separation & Disc area \\
\hline \multirow{2}{*}{ eOCT } & Mean (SD) & $165(72)$ & $179(77)$ & $295(43)$ & 21431 (1308) \\
\hline & Variance & 5147 & 5919 & 1868 & 1710562 \\
\hline \multirow{2}{*}{ cSLO } & Mean (SD) & 274 (188) & 295 (177) & 402 (179) & 20692 (3043) \\
\hline & Variance & 35393 & 31621 & 31891 & 9258368 \\
\hline \multirow{2}{*}{ Pearson's } & Correlation & -0.07 & 0.18 & -0.24 & -0.2 \\
\hline & $p$ Value & 0.7676 & 0.4591 & 0.3033 & 0.274 \\
\hline \multirow{2}{*}{ ANOVA } & $\mathrm{F}$ & 5.79 & 7.16 & 6.8 & 1.55 \\
\hline & p Value & 0.0211 & 0.0109 & 0.0129 & 0.218 \\
\hline
\end{tabular}

Figure 5 Comparison of eOCT and cSLO using data acquired in methods 1 (A) and 2 (B) with corresponding box plots (A, B) and descriptive statistics (C). The eOCT demonstrates a narrow range for all three parameters compared to the larger spread and high variance of the same parameters assessed by the cSLO. Whereas no correlation was found between eOCT and cSLO in any parameter measured (Pearson's correlation coefficient), there was a significant difference between the two imaging techniques for each parameter (ANOVA, $p<0.05$ ).

\section{Method 2: longitudinal slices and z-profiles}

The high depth resolution of eOCT is plainly apparent as two anatomical reflecting surfaces separated by approximate $300 \mu \mathrm{m}$ (50 frames) can be clearly distinguished (fig 2B, C). The transversal frames at the depth corresponding to the intensity maximum of each peak (fig 2D) clearly illustrate that the first peak arises from the retinal blood vessels (fig 2E) while the second peak arises from the scleral layer (fig $2 \mathrm{~F}$ ). Retinal thickness was not assessed.

By contrast with the eOCT, we were unable to demonstrate a double layer structure with cSLO images (fig 2G-K). 
Instead, a widely spread reflecting surface along the $\mathrm{z}$-axis is seen in the longitudinal slices (fig $2 \mathrm{H}, 2 \mathrm{I}$ ). Again, this is confirmed by the intensity z-profile, where there is only one peak with $600 \mu \mathrm{m}$ FWHM (10 frames, fig $2 \mathrm{~J}$ ). The transversal frame at the intensity maximum is illustrated in figure $2 \mathrm{~K}$.

\section{Method 2: multisurface depth analysis}

A histogram obtained from eOCT 3D images (fig 3A) has two clearly separated peaks corresponding to both reflective objects-that is, the retinal blood vessels and scleral layer. Compared to eOCT, the CSLO histogram of the same animal has wider, less distinct peaks (fig 3B). Double peak cSLO histograms were obtained only in 20 out of the possible 40 paired series of images.

Comparison between eOCT and cSLO histograms using the parameters of FWHM1, FWHM2, and the separation between peaks, demonstrates a narrower range for eOCT compared to the larger spread and high variance of the same parameters assessed by the cSLO. Again this is very much related to the poorer depth resolution of the cSLO, which makes estimates of tissue plane separation almost meaningless. There was no correlation between eOCT and CSLO in any parameter measured (fig 5C, Pearson's correlation coefficient). However, there was a significant difference between the two methods for each parameter (fig 5C, ANOVA, $\mathrm{p}<0.05$ ).

This method, unlike methods 1 and 3, does not rely on the subjective judgment of any parameter, as it provides complete objective data taken from the block of images acquired directly from the machine. Hence, it cannot be biased by observer interpretation.

\section{All methods: analysis of changes in ONH structure with IOP exposure}

All three methods were used to assess the ability of the eOCT to determine changes in the ONH with IOP exposure. Surgery produced an increase of IOP in all eyes with mean peak IOP elevation of $30.12(2.93) \mathrm{mm} \mathrm{Hg}$ and mean $\Delta \mathrm{IOP}$ integral of 615.56 (79.39) $\mathrm{mm} \mathrm{Hg}$ days at 3 months. The mean duration of IOP elevation was 57.39 (7.95) days.

Using method 1, we demonstrated progressive widening of the optic disc in both 3D reconstructions and $\mathrm{XY}$ projections over the 3 month period of study (fig 1). We found a significant correlation between the change in ODA and $\triangle \mathrm{IOP}$ integral (fig 6A, Pearson's $\mathrm{r}=0.564, \mathrm{p}<0.01$ ).

With the depth analysis histograms obtained using method 2, we found the parameter that changed most with IOP exposure in glaucomatous rats was "separation." This anatomically represents the "distance" between the retinal blood vessels and scleral layers. Figure 6B shows a significant correlation of "separation" with integral $\Delta \mathrm{IOP}$ (fig 6B, Pearson's $r=0.456, \mathrm{p}<0.05$ ). However we found no correlation between $\triangle \mathrm{IOP}$ integral and FWHMl or FWHM2.

Our analysis of method 3 showed interobserver and intraobserver coefficients of variation similar to those previously published for $\mathrm{HRT}^{20}{ }^{20}$ being 4.0 and 1.6, respectively. This method demonstrated progressive enlargement of the ASCO, representing the maximal opening diameter in the anterior surface of the sclera, in the same animals and in relation to integral $\Delta \mathrm{IOP}$. For example, in the animal shown in figure 1D-F, the ASCO measurements were $665 \mu \mathrm{m}$, $819 \mu \mathrm{m}$, and $919 \mu \mathrm{m}$ at baseline, 2 months and 3 months respectively, corresponding to the integral IOP of $0,542.65$, and 665.85 days. The duration of IOP elevation in this animal was 48 days with a peak elevation in IOP of $26 \mathrm{~mm} \mathrm{Hg}$. Progressive enlargement of the ASCO was significantly associated with integral $\Delta \mathrm{IOP}$ (fig 6C, Pearson's $r=0.462$, $\mathrm{p}<0.05$ ). Although we found a trend for the PSCO to increase with IOP exposure, this was not statistically significant. We
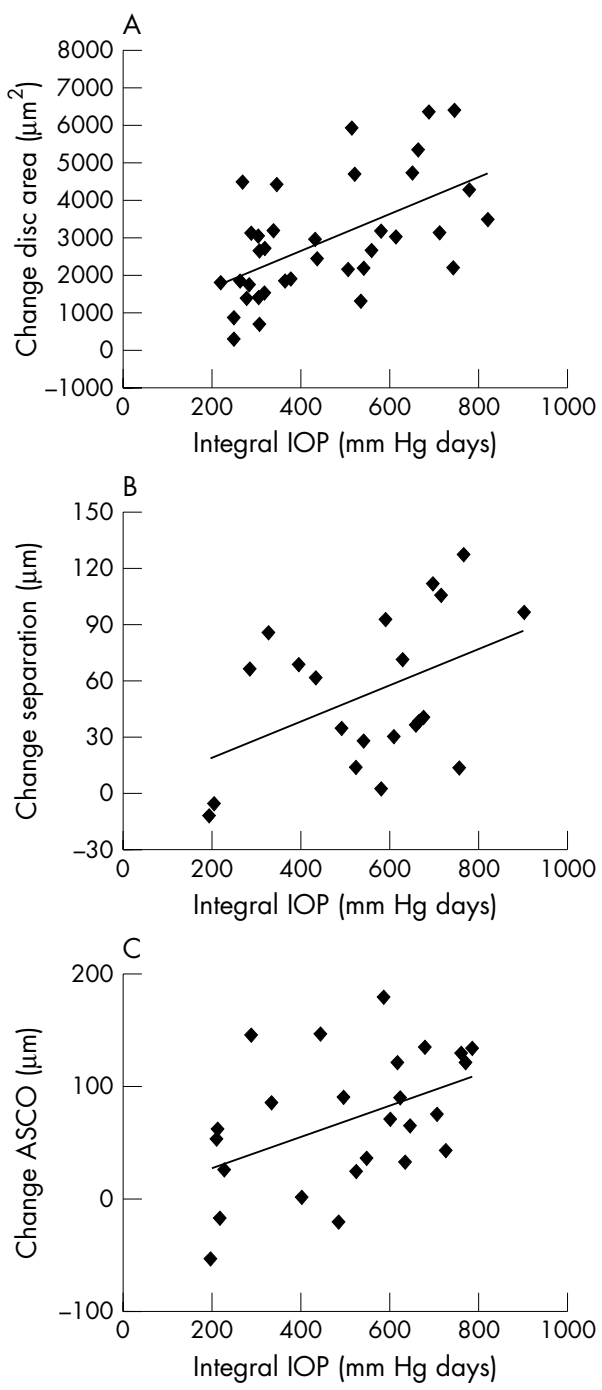

Figure 6 Analysis of changes in $\mathrm{ONH}$ structure with IOP exposure using eOCT. The change in disc area was found to be significantly correlated with $\triangle \mathrm{IOP}$ integral, (A, Method 1, Pearson's $r=0.546$, $p<0.01$ ), as was the degree of separation between the two anatomical layers, corresponding to the retinal blood vessel and the scleral layers ( $B$, Method 2, Pearson's $r=0.456, p<0.05$ ). Furthermore, we found a positive correlation between anterior scleral canal opening (ASCO) expansion and $\triangle \mathrm{IOP}$ integral $(C$, Method 3, Pearson's $r=0.462$, $\mathrm{p}<0.05$ ). All these results suggest that the eOCT can be used to objectively assess anatomical changes in $\mathrm{ONH}$ structure over time in glaucomatous rats.

found no correlation with the "thickness" of the scleral canal $($ " $\mathrm{t}$ ") and integral $\Delta \mathrm{IOP}$.

\section{DISCUSSION}

Objective assessment of the rat model of glaucoma has until recently not been possible. This is due to the small size of the rat eye and the poor depth resolution of currently available imaging techniques. ${ }^{11}$ We report the use of the new technique of eOCT in the in vivo investigation of $\mathrm{ONH}$ changes in rat glaucoma. Comparison of the eOCT with the cSLO shows the superiority of eOCT using a number of different methods, including a newly devised method of depth analysis that allows assessment of a multilayered anatomical structure. The multilayered structure of the rat ONH defined by the eOCT is consistent with our histology findings, as validated by confocal microscopy. Finally, using eOCT, we have 
analysed for the first time in vivo, changes in the scleral canal in rat glaucoma and their relation to IOP exposure.

Although fundus photography shows enlargement of the disc margin, suggesting also some enlargement of the scleral canal, it does not permit absolute quantification. ${ }^{21-23}$ Chauhan et al have recently advocated the use of the HRT for in vivo assessment of $\mathrm{ONH}$ changes in the rat model of glaucoma. ${ }^{11}$ However, the authors acknowledged that the limits of the depth resolution of the HRT and the complex structure of the rat $\mathrm{ONH}$ made the data, including volume assessment of the optic disc, difficult to interpret. ${ }^{11}$ There is a clear need therefore for an accurate imaging method to permit detailed analysis of changes at the rat $\mathrm{ONH}$ in vivo.

We employed eOCT, a new imaging technique, to assess structural changes in rat ONH with IOP exposure. Like HRT with CSLO, eOCT processes a series of transverse scans through the ONH. However, it is more powerful than HRT, as its depth resolution is 10 times higher, allowing for a larger number of slices to be acquired. Tissue surfaces are more clearly detected by the eOCT, allowing delineation of structures such as the scleral canal at the ONH. This is not achievable with HRT, and may explain why Chauhan and colleagues detected $\mathrm{ONH}$ cupping as a late feature. ${ }^{11}$ We suggest that as eOCT is a sensitive method of detecting structural changes in the $\mathrm{ONH}$, perhaps even before significant axonal loss has occurred, it may permit the early identification of disease processes.

The depth histogram method developed in this study allows a detailed depth analysis of multilayered structures. This is objective and parametric analysis which cannot be biased by observer variation. We have shown, using the parameters of FWHM1, FWHM2 and "separation," that measurements obtained by eOCT were consistently more accurate and with lower variance than the cSLO. This is to be expected since the high depth resolution of eOCT enables discrimination of two reflecting surfaces. This also explains why the spread of data, as illustrated by the variance factor, is considerable (at least six times greater) with cSLO compared to the eOCT. This high degree of variance also explains why we found no correlation between the two imaging techniques in depth analysis, and in fact showed a significant difference between them in the readings obtained.

Our data show that compared to the PSCO, expansion of the ASCO is positively correlated with IOP exposure. Although Chauhan and colleagues demonstrated scleral canal expansion with HRT, objective measurements of the ASCO and PSCO were not possible. ${ }^{11}$ As far as we are aware, this is the first time it has been possible to study these scleral canal parameters in vivo.

Burgoyne and colleagues have recently highlighted the ASCO as an important structural element in glaucoma. ${ }^{13}{ }^{14} 24-28$ Scleral canal expansion has also been identified as occurring in acute IOP elevation and in childhood glaucoma, being attributed to posteriorly directed stress forces at the $\mathrm{ONH}$ and increased compliance of young connective tissue. ${ }^{1314242930}$ This may be related to the fact that the lamina cribrosa (LC) is known to alter its biophysical properties with age, including an increase in stiffness and a decrease in reversal ability with age. ${ }^{31} 32$

The ASCO represents the edge of the LC-like structure in the rat $\mathrm{ONH}$, and like the human LC, this area is more vulnerable to IOP stress. ${ }^{33}{ }^{34}$ Our observations of IOP related deformation of anterior scleral canal support the clinical fact of early peripheral vision loss in glaucoma patients as a result of damage to the nerve fibres crossing the edge of the ONH. ${ }^{35}{ }^{36}$ The increased susceptibility of the ASCO compared to the PSCO may also be because of changes associated with pressure and depth, ${ }^{37}$ such that the anterior location of the ASCO would be expected to be maximally affected by IOP compared to the PSCO. Finally, expansion of the ASCO may be related to the effects of IOP on tissue compression/ stretching. Compression at the edge of the LC appears to increase as the lamina thins. ${ }^{33}$ Hence, the shear strain would be reached at lower IOPs in a "thin" compared to a "thick" LC. However we found no evidence of significant compression, as defined by scleral canal wall thickness (" $t$ ") in relation to IOP exposure in this study.

Precise measurements of $\mathrm{ONH}$ cupping or scleral canal expansion are an important prerequisite in experimental glaucoma, and there is a real need for objective data when assessing new therapies and strategies for this disease. The enhanced depth resolution of the eOCT compared to CSLO appears advantageous in assessing rat $\mathrm{ONH}$ to allow detailed structural analysis of the multilayered $\mathrm{ONH}$ structure. Burgoyne and co-workers have suggested that widening of the ASCO may be an early sign of glaucomatous damage. ${ }^{13} 14$ We propose that the eOCT has great potential in the early detection and diagnosis of glaucomatous disease in patients.

\section{ACKNOWLEDGEMENTS}

The authors would like to acknowledge Annelie Maass and Ted Garway-Heath.

Supported by The Wellcome Trust GR063658

AGhP and DAJ have received research funding from Ophthalmic Technologies Inc, Toronto Canada and The New York Eye and Ear Infirmary, USA.

\section{Authors' affiliations}

L Guo, V Tsatourian, V Luong, F W Fitzke, M F Cordeiro, Institute of Ophthalmology, London, UK

A G Podolean, D A Jackson, Applied Optics Group, School of Physical Sciences, University of Kent, Canterbury, UK

\section{REFERENCES}

1 Anderson DR. The management of elevated intraocular pressure with normal optic discs and visual fields. I. Therapeutic approach based on high risk factors. Surv Ophthalmol 1977;21:479-89.

2 Sommer A. Intraocular pressure and glaucoma. Am J Ophthalmol 1989; 107:186-8.

3 Radius RL, Pederson JE. Laser-induced primate glaucoma. II. Histopathology. Arch Ophthalmol 1984;102:1693-8.

4 Sawaguchi S, Yue BY, Fukuchi T, et al. Collagen fibrillar network in the optic nerve head of normal monkey eyes and monkey eyes with laser-induced glaucoma-a scanning electron microscopic study. Curr Eye Res 1999; 18:143-9.

5 Morrison JC, Dorman-Pease ME, Dunkelberger GR, et al. Optic nerve head extracellular matrix in primary optic atrophy and experimental glaucoma. Arch Ophthalmol 1990;108:1020-4.

6 Johnson EC, Morrison JC, Farrell S, et al. The effect of chronically elevated intraocular pressure on the rat optic nerve head extracellular matrix. Exp Eye Res 1996;62:663-74.

7 Pena JD, Agapova O, Gabelt BT, et al. Increased elastin expression in astrocytes of the lamina cribrosa in response to elevated intraocular pressure. Invest Ophthalmol Vis Sci 2001;42:2303-14.

8 Rogers J, Podoleanu A, Dobre G, et al. Topography and volume measurements of the optic nerve using en-face optical coherence tomography. Optics Express 2001;9:533-45.

9 Huang D, Swanson EA, Lin CP, et al. Optical coherence tomography. Science $1991 ; 254: 1178-81$

10 Hee MR, Puliafito CA, Wong C, et al. Quantitative assessment of macular edema with optical coherence tomography. Arch Ophthalmol 1995:113:1019-29.

11 Chauhan BC, Pan J, Archibald ML, et al. Effect of intraocular pressure on optic disc topography, electroretinography, and axonal loss in a chronic pressureinduced rat model of optic nerve damage. Invest Ophthalmol Vis Sci 2002;43:2969-76.

12 Hernandez MR, Pena JD. The optic nerve head in glaucomatous optic neuropathy. Arch Ophthalmol 1997; 115:389-95.

13 Bellezza AJ, Rintalan CJ, Thompson HW, et al. Anterior scleral canal geometry in pressurised (IOP 10) and non-pressurised (IOP 0) normal monkey eyes. Br J Ophthalmol 2003;87:1284-90.

14 Bellezza AJ, Rintalan CJ, Thompson HW, et al. Deformation of the lamina cribrosa and anterior scleral canal wall in early experimental glaucoma. Invest Ophthalmol Vis Sci 2003;44:623-37.

15 Morrison JC, Moore CG, Deppmeier LM, et al. A rat model of chronic pressure-induced optic nerve damage. Exp Eye Res 1997;64:85-96.

16 Cordeiro MF, Guo L, Luong V, et al. Real time imaging of single nerve cell apoptosis in retinal neurodegeneration. Proc Natl Acad Sci USA 2004; 101:13352-6. 
17 Guo L, Moss SE, Alexander RA, et al. Retinal ganglion cell apoptosis in glaucoma is related to intraocular pressure (IOP) and IOPinduced effects of extracellular matrix. Invest Ophthalmol Vis Sci 2005;46:175-82.

18 Fitzke F. Colour imaging using a scanning laser ophthalmoscope. Br J Ophthalmol 1998:82:337-8.

19 Naskar R, Wissing M, Thanos S. Detection of early neuron degeneration and accompanying microglial responses in the retina of a rat model of glaucoma. Invest Ophthalmol Vis Sci 2002;43:2962-8.

20 Garway-Heath DF, Poinoosawmy D, Wollstein G, et al. Interobserver and intraobserver variation in the analysis of optic disc images: comparison of the Heidelberg retina tomograph and computer assisted planimetry. Br J Ophthalmol 1999;83:664-9.

21 Sawada A, Neufeld A. Confirmation of the rat model of chronic, moderately elevated intraocular pressure. Exp Eye Res 1999;69:525-31.

22 Cohan BE, Pearch AC, Jokelainen PT, et al. Optic disc imaging in conscious rats and mice. Invest Ophthalmol Vis Sci 2003;44:160-3.

23 Neufeld A, Sawada A. Inhibition of nitric-oxide synthase 2 by aminoguanidine provides neuroprotection of retinal ganglion cells in a rat model of chronic glaucoma. Proc Natl Acad Sci USA 1999:96:9944-8.

24 Robin AL, Quigley HA, Pollack IP, et al. An analysis of visual acuity, visual fields, and disk cupping in childhood glaucoma. Am J Ophthalmol 1979;88:847-58.

25 Weinreb RN, Dreher AW, Bille JF. Quantitative assessment of the optic nerve head with the laser tomographic scanner. Int Ophthalmol 1989:13:25-9.

26 Weinreb RN, Lusky M, Bartsch DU, et al. Effect of repetitive imaging on topographic measurements of the optic nerve head. Arch Ophthalmol 1993; 111:636-8.

27 Brigatti L, Weitzman M, Caprioli J. Regional test-retest variability of confocal scanning laser tomography. Am J Ophthalmol 1995; 120:433-40.
28 Zangwill L, Shakiba S, Caprioli J, et al. Agreement between clinicians and a confocal scanning laser ophthalmoscope in estimating cup/disk ratios. Am J Ophthalmol 1995; 119:415-21.

29 Quigley HA, Hohman RM, Addicks EM, et al. Morphologic changes in the lamina cribrosa correlated with neural loss in open-angle glaucoma. Am J Ophthalmol 1983;95:673-91.

30 Jonas JB, Gusek GC, Naumann GO. Optic disc morphometry in chronic primary open-angle glaucoma. I. Morphometric intrapapillary characteristics. Graefes Arch Clin Exp Ophthalmol 1988;226:522-30.

31 Albon J, Purslow PP, Karwatowski WS, et al. Age related compliance of the lamina cribrosa in human eyes. Br J Ophthalmol 2000;84:318-23.

32 Albon J, Karwatowski WS, Easty DL, et al. Age related changes in the noncollagenous components of the extracellular matrix of the human lamina cribrosa. Br J Ophthalmol 2000;84:311-17.

33 Edwards ME, Good TA. Use of a mathematical model to estimate stress and strain during elevated pressure induced lamina cribrosa deformation. Curr Eye Res 2001;23:215-25.

34 Mathematical modelling of the effect of elevated intraocular pressure on the lamina cribosa. 16th ASCE Engineering Mechanics Conference. Seattle: University of Washington, 16-18 July, 2003.

35 Minckler DS. The organization of nerve fiber bundles in the primate optic nerve head. Arch Ophthalmol 1980;98:1630-6.

36 Quigley HA, Addicks EM. Regional differences in the structure of the lamina cribrosa and their relation to glaucomatous optic nerve damage. Arch Ophthalmol 1981;99:137-43.

37 Morgan WH, Yu DY, Alder VA, et al. The correlation between cerebrospinal fluid pressure and retrolaminar tissue pressure. Invest Ophthalmol Vis Sci 1998;39:1419-28. 


\section{PostScript}

\section{LETTERS}

\section{Bilateral naevus of Ota with choroidal melanoma and diffuse retinal pigmentation in a dark skinned person}

Naevus of Ota (naevus fusculocoeruleus ophthalmomaxillaris) was described by the Japanese dermatologist, Ota, in 1939 as a dermal melanocytic hamartoma that presents as bluish hyperpigmentation along the ophthalmic, maxillary, and mandibular branches of the trigeminal nerve. It is bilateral in less than 5\% cases, occurring frequently in Orientals $(0.2 \%-1 \%)$ and darker races and rarely in white people $(0.04 \%)$. Open angle glaucomas and choroidal melanoma are the rare ocular involvements. Ota's naevus is more common in Asians than white people but uveal melanoma occurs predominantly in white populations. ${ }^{12}$ Dark skinned patients represent only $1 \%$ of all cases of orbital melanomas. ${ }^{3}$ The risk of developing uveal melanoma in a patient with naevus of Ota is one in 400 patients in their lifetime. ${ }^{1}$ We report a rare case of bilateral naevus of Ota with a right (RE) choroidal melanoma and left (LE) diffuse pigmentation of retina.

\section{Case report}

A 73 year old Anglo-Indian woman was referred with complaints of photopsia. She had black hair and light brown skin. Examination revealed a brownish-black pigmentation of the conjunctiva, episclera, and periocular skin bilaterally (fig 1). Visual acuity for distance and near was $6 / 6$ and N5, respectively, in each eye. Heterochromia was present, the right iris being a darker brown than the left, which had a sector of light brown colour. Gonioscopy and intraocular pressure were normal. The right fundus revealed a pigmented, large, elevated choroidal mass 10 disc diameter (DD) in size, 4 DD superonasal to the disc. Drusen were overlying it. No subretinal fluid was seen. The left eye showed a patchy dark pigmentation 3 DD in size, at the temporal edge of the macula. A ridge-like pigmented elevation, 3 DD long, was also seen along the superonasal vessels. Both optic discs and maculas were normal. Ultrasound in the right eye showed a $10 \mathrm{~mm}$ tumour, $4.2 \mathrm{~mm}$ high. Fluorescein angiography confirmed its independent circulation. A systemic examination found no signs of metastasis. A diagnosis of a

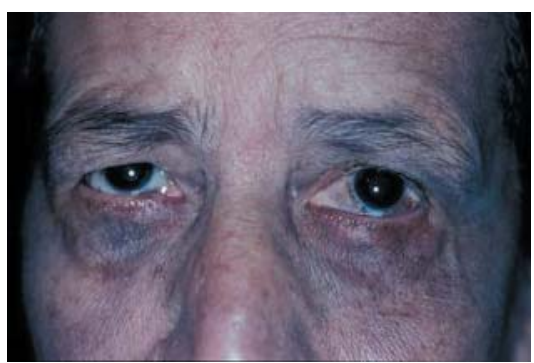

Figure 1 Oculodermal pigmentation.

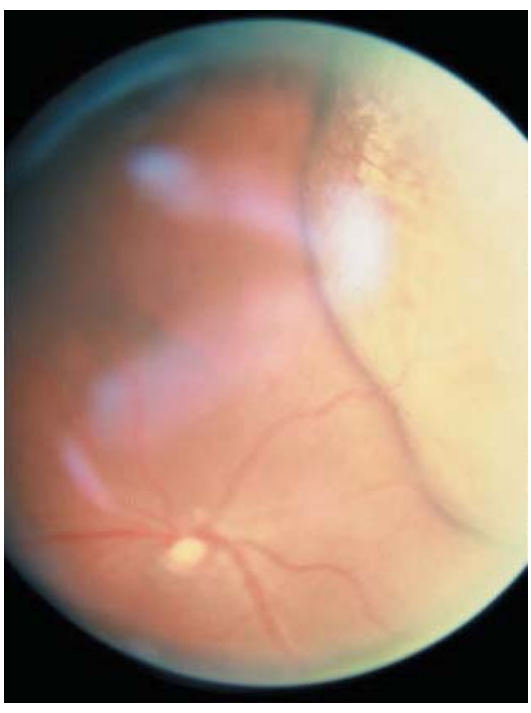

case illustrates the need for regular ophthalmic review of all pigmented lesions and the recognition that patients with naevus of Ota may also have the additional complication of melanoma. There is need for close observation of all pigmented lesions of the eye. Regardless of the patient's race, there is a greater than normal chance that a patient with the naevus of Ota might have a malignant melanoma develop within one of the affected tissues.

S Sharan, J R Grigg, F A Billson Save Sight Institute, University of Sydney, Department of Ophthalmology, Sydney Eye Hospital, 8 Macquarie Street, Sydney 2000, Australia

Correspondence to: Sapna Sharan, Save Sight Institute, University of Sydney, Department of Ophthalmology, Sydney Eye Hospital, 8 Macquarie Street, Sydney 2000, Australia; sapnasd@yahoo.co.uk

doi: 10.1136/bjo.2005.070839

Accepted for publication 1 May 2005

Figure 2 Choroidal melanoma.

bilateral naevus of Ota with low grade, choroidal melanoma in the right eye (fig 2) and retinal pigmentation in the left eye was made. The patient was reluctant to accept the option of enucleation in view of the right vision. A ${ }^{125}$ I radioactive plaque was applied (COMS study). A follow up examination 3 years postoperatively showed a flatter, yellow $4 \mathrm{DD} \times 1.5 \mathrm{DD}$ scarred tumour with mottled pigmentation. The left melanosis remained unchanged. The vision was $6 / 6$ in both eyes 6 years after ${ }^{125} \mathrm{I}$ treatment and cataract surgery.

\section{Comment}

Ota's naevus is commonly seen unilaterally $(90 \%)$. Bilateral involvement is rare. It represents melanocytes that have not migrated completely from the neural crest to the epidermis during the embryonic stage. Orientals and pigmented races have a high prevalence with a predilection for women (1: 4.8). Variable prevalence among different populations suggests genetic influences, although familial cases are rare. Two peak ages of onset in early infancy (50\%) and in early adolescence suggest hormonal influence. ${ }^{1}$ In addition to the skin, pigmentation may involve oral mucosa, tympanic membrane, intranasal mucosa, leptomeninges and ocular structures such as the sclera, retrobulbar fat, cornea, lens, trabeculum, disc, and retina. Associated malignant melanomas of the uvea, orbit, skin, and CNS have been described. ${ }^{2}$ Choroidal melanomas are known to occur in less than $4 \%$ of cases and glaucoma has been noticed in less than $10 \%$ of cases. ${ }^{4}$

Our case reports a rare occurrence of bilateral naevus of Ota with choroidal malignant melanoma in the right eye and retinal pigmentation in the left eye in a pigmented person. She was born to Anglo-Indian parents but did not know how far back in time the intermarriage had occurred. Ophthalmological follow up care is necessary for patients with increased melanosis. This

\section{References}

1 Hidano A, Kajima H, lkeda S, et al. Natural history of nevus of Ota. Arch Dermatol 1967;95:187-95.

2 Gonder JR, Shields JA, Albert DM, et al. Uveal malignant melanoma associated with ocular and oculodermal melanocytosis. Ophthalmology 1982;89:953-60.

3 Margo CE, McLean IW. Malignant melanoma of the choroid and ciliary body in black patients. Arch Ophthalmol 1984;102:77-9.

4 Roy PE, Schaeffer EM. Nevus of Ota and choroidal melanoma. Surv Ophthalmol 1967; 12:130-40.

\section{Treatment of neurotrophic keratopathy with nasal dilator strips}

Neurotrophic keratopathy, characterised by poorly healing corneal epithelium, occurs in eyes with decreased corneal sensory innervation. Clinical findings include chronic epithelial defects and corneal ulceration. Numerous conditions predispose to neurotrophic keratopathy including diabetes mellitus, accidental and surgical trauma, herpes simplex and herpes zoster keratitis, leprosy, and topical anaesthetic abuse.

Management of neurotrophic keratopathy includes ocular lubrication, pressure patching, autologous serum eye drops, ${ }^{1}$ fitting of a bandage contact lens, ${ }^{2}$ amniotic membrane grafting, ${ }^{34}$ and surgical tarsorrhaphy. Surgical tarsorrhaphy can be very successful in resolving neurotrophic corneal ulceration, ${ }^{5}$ but many patients find this option cosmeti-

We describe a novel method of nonsurgical tarsorrhaphy using over the counter adhesive, non-medicated, nasal dilator strips (NDS) (Breathe Right Nasal Strips, Whippany, NJ, USA) applied vertically across the eyelids (fig 1). The adhesive strip consists of parallel bands of plastic imbedded in a pad, and is available in different sizes.

The nasal strips were originally developed to treat patients with snoring problems, ${ }^{6}$ or to improve nasal congestion. ${ }^{7}$ In rhinological applications, the strip is typically used cally unacceptable. 


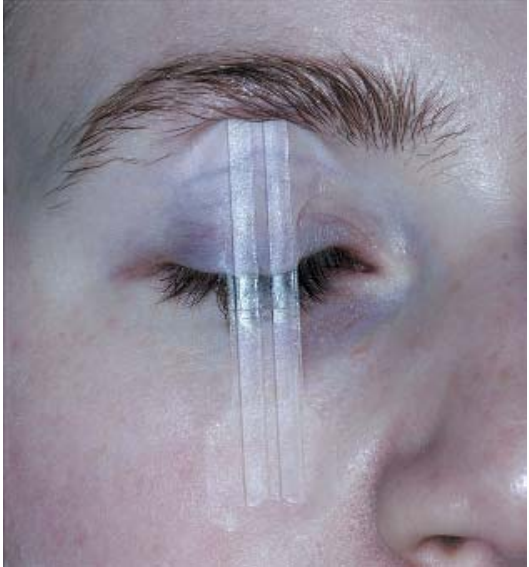

Figure 1 Applying a nasal dilator strip vertically over the eyelid creates an easily reversible tarsorrhaphy. It also provides an effective and, for patients, cosmetically acceptable way to treat chronic corneal neurotrophic disorders.

horizontally across the nose in order to open the nasal airway. In the current study, we applied the strip vertically over the closed eyelid as shown in figure 1 . The adhesive strip creates a firm and effective eyelid closure, and patients can control the application and removal of the strip. The strips have the advantage of being relatively inexpensive, reusable, and reversible, and their use has replaced standard eye patching in our clinical practice. We have noted success with the use of these strips for the management of neurotrophic ulceration and describe two representative cases.

\section{Case reports}

A 60 year old woman developed a neurotrophic corneal ulcer following a complicated retinal detachment repair. After a year of standard medical therapies, including lubrication and frequent conventional patching, she continued to have a $4 \mathrm{~mm} \times 4 \mathrm{~mm}$ chronic non-healing epithelial defect. Treatment with reversible NDS tarsorrhaphy was initiated with instructions to apply the strips at bedtime and as much as possible during the day. Nine weeks later the corneal epithelial defect had healed completely. Over the next year she gradually decreased the wearing time of the strips and is currently stable without their use.

A 48 year old woman with a $6 \mathrm{~mm} \times 2 \mathrm{~mm}$ neurotrophic corneal ulcer was referred for management after failing numerous medical and surgical therapies including lubrication, autologous serum eye drops, patching, and an amniotic membrane graft. The patient was instructed to use NDS tarsorrhaphy according to the schedule described in the previous case. Within 2 weeks the corneal epithelial defect healed completely. The patient continues to apply the tarsorrhaphy but with decreasing frequency.

The novel use of nasal dilator strips to perform a temporary tarsorrhaphy has aided us greatly in our management of neurotrophic corneal ulceration. We believe it is an attractive, cost effective, efficient alternative to patching for any ocular condition. In addition, nasal strip tarsorrhaphy allows for immediate reversibility that facilitates patient acceptance.
M T Magone, G D Seitzman, S Nehls, T P Margolis Francis I Proctor Foundation, University of California San Francisco, 95 Kirkham Street, San Francisco, CA 94143, USA

Correspondence to: T P Margolis, MD, PhD, Francis I Procter Foundation, 95 Kirkham Street, San Francisco, CA 94143, USA; tpms@itsa.ucsf.edú doi: 10.1136/bjo.2005.073114 Accepted for publication 10 May 2005

\section{References}

1 Matsumoto Y, Dogru M, Goto E, et al. Autologous serum application in the treatment of neurotrophic keratopathy. Ophthalmology

2004;111:1115-20.

2 Montero J, Sparholt J, Mely R, et al. Retrospective case series of therapeutic applications of lotrafilcon a silicone hydrogel soft contact lenses. Eye Contact Lens 2003;29:72-5.

3 Solomon A, Meller D, Prabhasawat $\mathrm{P}$, et al. Amniotic membrane grafts for nontraumatic corneal perforations, descemetoceles, and deep ulcers. Ophthalmology 2002; 109:694-703.

4 Prabhasawat $\mathbf{P}$, Tesavibul N. Preserved amniotic membrane transplantation for conjunctival surface reconstruction. Cell Tissue Bank 2001;2:31-9.

5 Cosar CB, Cohen EJ, Rapuano CJ, et al. Tarsorrhaphy: clinical experience from a cornea practice. Cornea 2001:20:787-91.

6 Ufberg J. Fenton G. Effect of breathe right nasal strip on decrease snoring. Rhinology 1997:35:50-2.

7 Ochi K, Mitsui M, Kaneko T, et al. The application of Breathe Right for otorhinolaryngologic disorders. Otorhinology 1997;90:597-601.

\section{Confocal microscopy of the cornea in nephropathic cystinosis}

Cystinosis is an autosomal recessive inherited disorder of amino acid metabolism characterised by the deposition of cystine crystals in the eye, kidney, reticuloendothelial system, and various other tissues. ${ }^{1}$ Childhood or nephropathic cystinosis can present as an infantile or a juvenile variant. ${ }^{1}$ The infantile variant tends to have a more devastating course and is associated with growth retardation, rickets, and eventual renal failure which requires transplantation within the first decade. ${ }^{1}$ The juvenile variant has later onset and milder nephropathy.

In nephropathic cystinosis, crystal deposits usually appear in the peripheral, anterior cornea within the first year of life and progress centrally and posteriorly until the entire cornea is involved..$^{2-7}$ The diagnosis can be confirmed histopathologically by demonstration of characteristic crystals by electron microscopy in a conjunctival biopsy. ${ }^{89}$ Stromal deposition of crystal deposits has been demonstrated by confocal microscopy. ${ }^{9}$ We provide the first demonstration, to the best of our knowledge, of cystine crystals in the corneal epithelium using in vivo confocal microscopy.

\section{Case report}

A 9 year old boy presented to the King Khaled Eye Specialist Hospital in Riyadh, Saudi Arabia, with a complaint of recurrent foreign body sensation, associated with severe photophobia and blepharospasm. He had been diagnosed with infantile nephropathic cystinosis at age of 9 months and had been treated with systemic cysteamine. On examination, the visual acuity was $20 / 20$ in the right eye and $20 / 25$ in the left eye. The

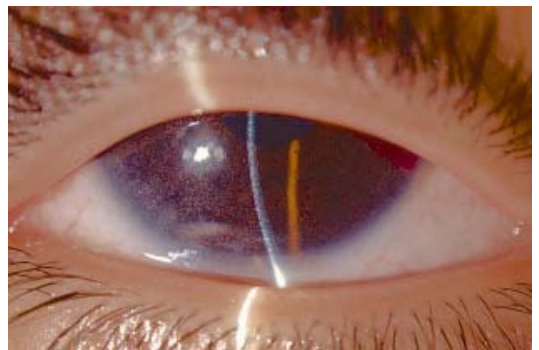

Figure 1 Crystal deposits in the right eye predominantly involving the anterior and midstroma, with limbus to limbus distribution.

intraocular pressure was $12 \mathrm{~mm} \mathrm{Hg}$ in both eyes. Slit lamp examination showed crysta deposits of 2.5 in Gahl density score ${ }^{7}$ in both corneas, predominantly involving the anterior stroma and with limbus to limbus distribution (fig 1). Dilated fundus examination was normal with no maculopathy or peripheral retinal pigment abnormalities Topical treatment with cysteamine $0.5 \%$ drops resulted in symptomatic relief.

Confocal microscopy (Confoscan 3, Nidek Technologies, Vigonza, Italy) demonstrated crystalline deposits in the corneal epithelium (fig 2A, B) and stroma (fig 2C, D). Crystal deposits in the corneal epithelium were needle shaped and fusiform shaped and oriented parallel to the plane of the epithelial cells (fig 2A, B). In the basal cell layer, the crystals were associated with dendritic cells (fig 2B). The highest crystal density was in the mid-stroma, where fusiform shaped crystals were more predominant than needle shaped crystals (fig 2C). The lowest crystal density was in the posterior stroma, where most of the deposits were needle shaped (fig 2E). Within the stroma the crystals were oriented parallel to the plane of the stromal lamella. The needle shaped crystals were highly variable in length with some as long as $100 \mu \mathrm{m}$. The endothelial cell layer was normal.

\section{Comment}

The current case clearly documents that crystalline deposits may be found in the epithelium of patients with nephropathic cystinosis, unlike previous electron microscopic $^{8}$ and confocal microscopic ${ }^{9}$ studies that suggest these deposits are localised to the stroma. In addition, we found maximum crystal density in the mid-stroma and minimum density in the posterior stroma, in contrast with a previous report in which maximum crystal density was just anterior to Descemet's membrane. ${ }^{9}$

We hypothesise the presence of these abnormal deposits in the corneal epithelium may contribute, in part, to the foreign body sensation and photophobia that is invariably associated with this disorder, as well as the predisposition to recurrent epithelial erosions. Chronic low grade inflammation of the epithelium and epithelial basement membrane zone associated with recurrent epithelial erosions is the probable explanation for the presence of dendritic cells in the basal epithelium of the central cornea. ${ }^{10}$ Successful reduction in the density of corneal crystals and symptomatic relief was obtained with the use of topical cysteamine $0.5 \%$ drops, as in previous reports. ${ }^{5-7}$ 

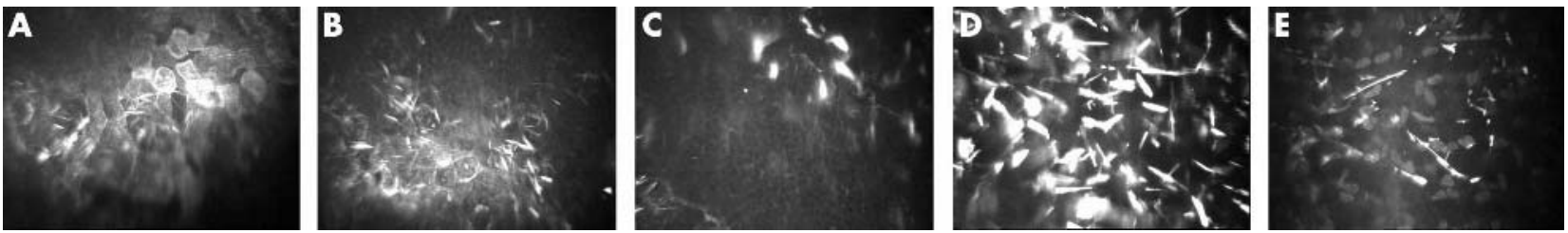

Figure 2 Crystal deposits in the corneal epithelium and stroma. A mixture of needle shaped and fusiform shaped crystals are present in (A) the superficial epithelial cell layer and (B) the wing cell layer. (C) Dendritic cells are present in the basal cell layer. (D) The greatest density of crystals is in the mid-stroma, where fusiform shaped crystals are the predominant morphology. (E) The least density of crystals is in the posterior stroma, where needle shaped crystals are the predominant morphology.

A H Alsuhaibani, M D Wagoner Anterior Segment Division, Department of Ophthalmology, King Khaled Eye Specialist Hospital, Riyadh, Kingdom of Saudi Arabia

A O Khan

Pediatric Ophthalmology and Strabismus Division, Department of Ophthalmology, King Khaled Eye Specialist Hospital, Riyadh, Kingdom of Saudi Arabia

Correspondence to: Michael D Wagoner, MD, King Khaled Eye Specialist Hospital, PO Box 7191, Riyadh 11462, Kingdom of Saudi Arabia; mwagoner@kkesh.med.sa

doi: 10.1136/bjo.2005.074468

Accepted for publication 10 May 2005

Competing interests: none declared

\section{References}

1 Theone JG. Cystinosis. J Inherit Metab Dis 1995; 18:380-6.

2 Wong VG, Schulman JD, Seegmiller JE. Conjunctival biopsy for the biochemical diagnosis of cystinosis. Am J Ophthalmol 1970;70:278-81.

3 Kaiser-Kupfer MI, Chan CC, Rodriques M, et al. Nephropathic cystinosis: immunohistochemical and histopathologic studies of cornea, conjunctiva, and iris. Curr Eye Res 1987:66:17-22.

4 Cotran PR, Bajart AM. Congenital corneal opacities. Int Ophthalmol Clin 1992;32:93-105.

5 Iwata F, Kuehl EM, Reed GF, et al. A randomized clinical trial of topical cysteamine disulfide (cystamine) versus free thiol (cysteamine) in the treatment of corneal cystine crystals in cystinosis. Mol Genet Metab 1998;64:237-42

6 Gahl WA, Kuehl EM, Iwata F, et al. Corneal crystals in nephropathic cystinosis: natural history and treatment with cysteamine eyedrops. Mol Genet Metab 2000;71:100-20.

7 Khan AO, Latimer B. Successful use of topical cysteamine formulated from the oral preparation in a child with keratopathy secondary to cystinosis. Am J Ophthalmol 2004;138:674-5.

8 Kenyon KR, Sensenbrenner JA. Electron microscopy of cornea and conjunctiva in childhood cystinosis. Am J Ophthalmol 1974;78:68-76.

9 Grupcheva CN, Ormonde SE, McGhee C. In vivo confocal microscopy of the cornea in nephropathic cystinosis. Arch Ophthalmol 2002; 120:1742-5.

10 Gillette TE, Chandler JW, Greiner JV. Langerhans cells of the ocular surface. Ophthalmology 1982;89:700-11.

Total parenteral nutrition, vitamin $E$, and reversible macular dysfunction morphologically mimicking age related macular degeneration

A variety of nutrient deficiencies may predispose to the development of age related macular degeneration (AMD). ${ }^{1}$ Patients receiving parenteral nutrition (TPN) may be at particular risk of early onset AMD, because of inadequate or excess nutritional supplementation. ${ }^{1} \quad$ Studies including the Eye Disease Case-Control Study and Beaver Dam Eye Study have evaluated the relation between antioxidant and micronutrient levels, and the risk of AMD. ${ }^{2-4}$ A protective effect of high plasma vitamin E levels was convincingly demonstrated. ${ }^{5}$

We describe a patient treated with parenteral fluid support who developed visual symptoms and signs of AMD, in conjunction with longstanding vitamin E deficiency. Isolated cases of visual disturbance in patients undergoing TPN have been reported in the literature ${ }^{67}$; however, to our knowledge, no case of visual disturbance attributed to vitamin E deficiency has been reported in this context.

\section{Case report}

A 57 year old man received parenteral fluid five times a week at home because of short bowel syndrome secondary to Crohn's disease. It was thought he had undergone bowel adaptation to meet macronutrient and micronutrient needs in the 13 years since his surgery. He presented with subacute visual disturbance. He described altered colour perception in situations analogous to macular stress testing (moving from dark adapted situations to bright lights) and enlarging central scotomata. Visual acuity was $6 / 6$ in the right eye, 6/12 in the left. Visual fields, intraocular pressures, and neurological examination were normal. Funduscopy revealed macular soft drusen, and extensive subretinal basal laminar deposits in the macular region, more marked in the right than left eye (fig 1). Electroretinogram was normal.

The patient was receiving electrolyte support 6 days a week at time of presentation. Measured haematological parameters and

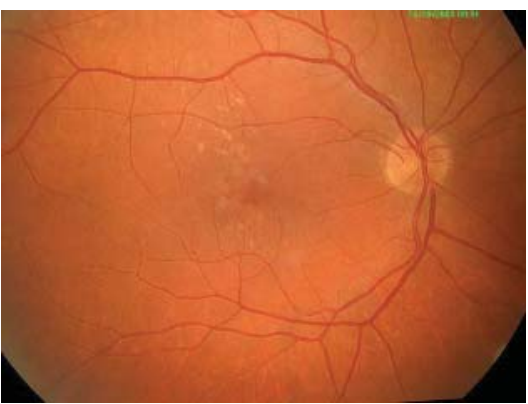

Figure 1 Macular soft drusenosis, characterised by subretinal basal laminar deposits in the macular region. urea and electrolyte levels revealed a low haemoglobin level $(11.0 \mathrm{~g} / \mathrm{dl})$, and a mild degree of macrocytosis (102.3 fl). Because hypervitaminosis and/or deficiency in trace minerals were suspected, serum values of vitamins $\mathrm{A}, \mathrm{E}, \mathrm{B} 1, \mathrm{~B} 2, \mathrm{~B} 6$, plasma zinc, copper, selenium, manganese, caeruloplas min, and red cell GSH activity were measured. Results revealed vitamin E deficiency ( $12 \mu \mathrm{mol} / \mathrm{l}$, normal range: $14-39 \mu \mathrm{mol} / \mathrm{l}$ ). A retrospective survey of previous serum vitamin E levels suggested longstanding deficiency, with levels of $10 \mu \mathrm{mol} / \mathrm{l}$, and $13 \mu \mathrm{mol} /$ l, 6 months and 1 year respectively, before onset of symptoms. Treatment with vitamin supplementation lead to complete resolution of symptoms in 3 weeks. Vitamin E levels returned to normal; however, fundal appearances remained unchanged.

\section{Comment}

The presence of bilateral hard and soft drusen and pigmentary abnormalities in the macula are the clinical hallmarks of AMD. ${ }^{8}$ The early onset of morphological changes at Bruch's membrane/retinal pigment epithelium (RPE) interface may relate to vitamin or micronutrient deficiency, associated with parenteral nutrition. ${ }^{7}$

Cumulative oxidative damage may have an important role in the pathogenesis of AMD, since accumulation of lipofuscin pigments may arise as a consequence of antioxidant deficiency, or under pro-oxidant conditions. Evidence exists for an association between atrophic AMD and excessive lipofuscin accumulation. ${ }^{89}$ Compromised RPE in this context is believed to be due to the amphiphilic structure and photoreactivity of the di-retinal conjugate A2E, the major constituent of lipofuscin. ${ }^{59}$ Antioxidant vitamins have been shown to aid in the defence against AMD. Vitamins E and C suppress A2E epoxidation, suggesting one mechanism by which these vitamins may protect the ageing macula.

Vitamin E deficiency was present consistently over the 12 month period preceding symptom onset, reinforcing the likelihood that the clinical presentation had been caused by vitamin E deficiency. Vitamin E deficiency results in retinal degeneration, excessive RPE lipofuscin, and decrease in the polyunsaturated fatty acid content of rod outer segments and the RPE. ${ }^{5}$ Furthermore, vitamin E deficiency may cause mild macrocytic anaemia and accumulation of ceroid lipofuscin in nerves, affecting function of central and peripheral nervous systems. ${ }^{6}$ Patients with sufficient gut length for protein calorie nutrition receiving parenteral fluids may run the risk of micronutrient deficiency despite a normal diet, and may present to the ophthalmology department. We recommend formal micronutrient screening in patients with extensive small bowel resection. 
L Porter

Dundee Medical School, Dundee, UK

N Reynolds

Department of Medicine, Dundee Medical School,

Dundee, UK

J D Ellis

Department of Ophthalmology Ninewells Hospital and Medical School, Ninewells Road, Dundee DD1 9SY,

Correspondence to: Dr John Ellis, Department of Ophthalmology, Ninewells, Dundee DDI 9SY, UK; john.ellis@tuht.scot.nhs.uk

doi: 10.1136/bjo.2005.074195

Accepted for publication 23 May 2005 Funding: none.

Conflict of interest: none.

\section{References}

1 Vinton NE. Heckenlively JR, Laidlaw SA, et al. Visual function in patients undergoing long-term total parenteral nutrition. Am J Clin Nutr 1990:52:895-902.

2 The Eye Disease Case-Control Study Group. Antioxidant status and neovascular age-related macular degeneration. Arch Ophthalomol 1993;111:1366, 1499, and 104-9.

3 Lyle BJ, Mares-Perlman JA, Klein BEK, et al. Antioxidant intake and risk of age-related nuclear cataracts in the Beaver Dam Eye Study. Am J Epidemiol 1999; 149:801-9.

4 Seddon JM, Ajani UA, Sperduto RD, et al. Dietary carotenoids, vitamins $A, C$ and $E$, and advanced age-related macular degeneration. JAMA 1994;272: 1413-20.

5 Beatty S, Hui-Hang K, Henson D, et al. The role of oxidative stress in pathogenesis of age-related macular degeneration. Surv Ophthalmol 2000;45: 115-34

6 Kawakubo K, Matsumoto T, Mochizuki Y, et al. Progressive encephalopathy in a Crohn's disease patient on long-term total parenteral nutrition: possible relationship to selenium deficiency. Postgrad Med J 1994;70:215-9.

7 Yassur Y, Snir M. Melamed S. Bilateral maculopathy simulating "cherry-red spot" in a patient with Crohn's disease. Br J Ophthalmol 1981;65:184-8.

8 Zurdel J, Richard G. Clinical manifestations and natural history and ARMD. ARMD Current

Treatment Concepts. Medical radiology. London: Springer, 2001

9 Sparrow JR, Fishkin N, Zhou J, et al. A2E a byproduct of the visual cycle. Vis Res 2003;43:2983-90.

\section{Spontaneous involution of autologous lenses and phacoanaphylaxis reaction in Stickler syndrome}

Stickler syndrome is a "hereditary progressive arthro-ophthalmopathy"1 caused in the majority of cases by mutations of the COL2AI gene encoding for type II collagen. ${ }^{2}$ The disease is transmitted as an autosomal dominant trait with high penetrance but variable expressivity. ${ }^{3}$ Most common ocular manifestations of the disease are myopia, vitreous veils and degeneration, early cataract, retinal peripheral breaks and retinal detachment. $^{34}$

\section{Case report}

This patient had typical ocular and extraocular clinical manifestations of Stickler syndrome. She was fitted with contact lenses $(-17.00$ dioptres $)$ at the age of 1 month. Despite the relatively poor vision, hearing
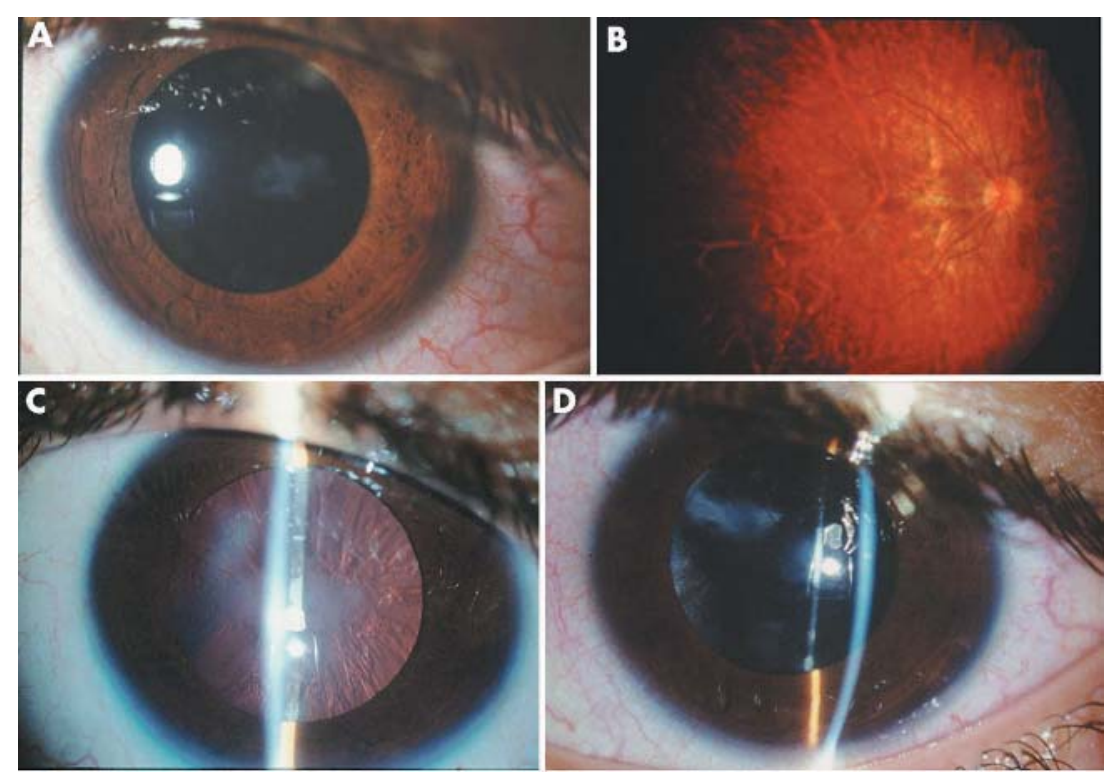

Figure 1 (A) Mild opacity of the lens posterior capsule of the right eye initially observed at the age of 7 years. (B) Appearance of the fundus demonstrating the retinal pigmentary changes in the periphery and retinal degenerative changes within the posterior pole. (C) Marked opacification and fusion of the lens capsules in the right eye observed at 9 years of age. (D) Mild capsule opacities are still observed 2 years later, at 11 years of age. The refractive error at this stage is +1.25 and the visual acuity for distance is $6 / 12(20 / 40)$

impairment and skeletal problems, she developed well mentally and attended regular school. With glasses $(-15.00)$ the visual acuity (VA) was stable, around 6/21 (20/75) for distance and $\mathrm{J} 2$ for near in both eyes.

A mild central opacity of the posterior lens capsule was initially observed in both eyes

when she was 7 years old (fig 1A). The IOP was $12 \mathrm{~mm} \mathrm{Hg}$, the corneas clear, anterior chambers deep and devoid of any inflammatory signs. Fundus examination disclosed no changes from previous examinations (fig lB) Refraction and VA in both eyes remained unchanged.
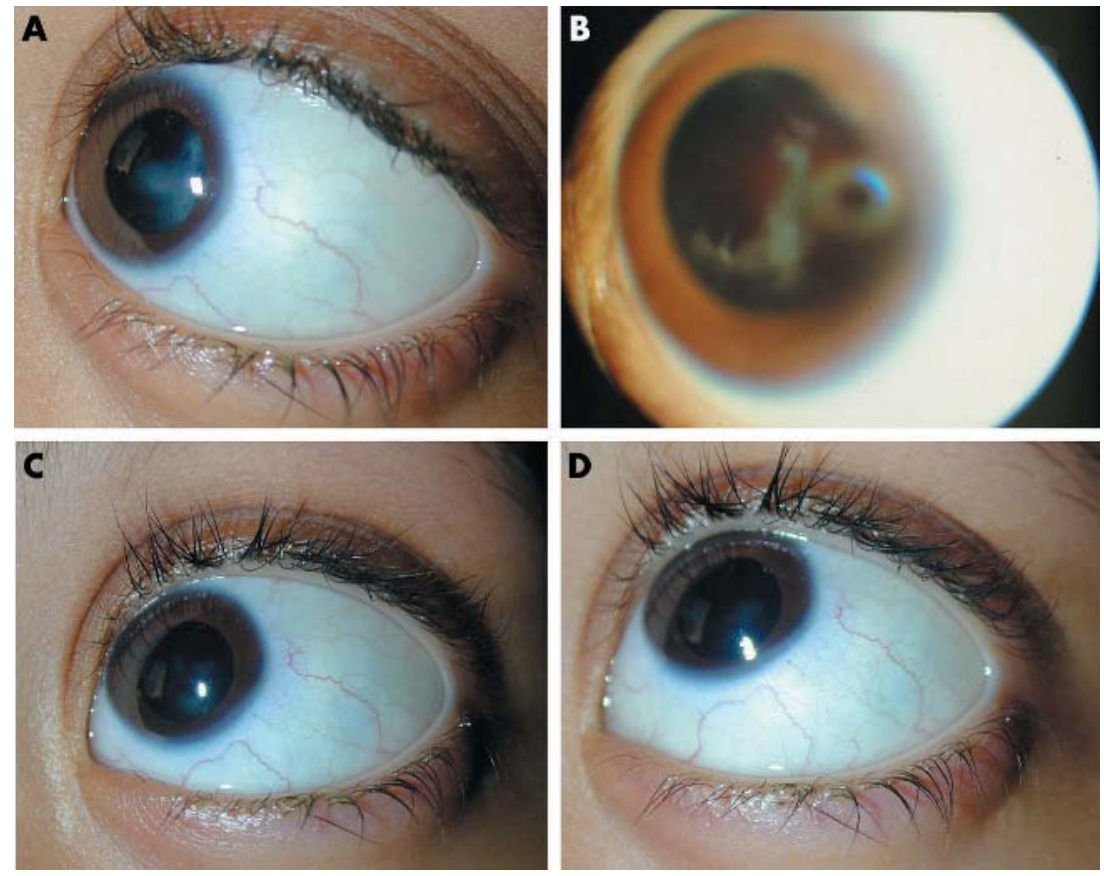

Figure 2 (A) Large cortical remnants are seen within the capsular bag remnant in the left eye with an intense flare and many inflammatory cells. The cornea is still mildly hazy 2 weeks after the phacoanaphylactic reaction. (B) The vitreous of the left eye is hazy with many cortical lens remnants observed with transillumination at the slit lamp. (C) Right eye is quiet, the refractive error is +1.25 and the uncorrected visual acuity $6 / 12(20 / 40)$ despite the presence of mild lens capsule opacities. (D) Left eye is also quiet showing the same characteristics as the right eye. 
On 23 June 2002, at the age of 9 years, she complained of blurred vision in the right eye. Without glasses, VA for distance was 6/60 $(20 / 200)$ and for near less than J16. Involution of the lens material with marked opacity of the fused capsules was detected (fig lC). Accurate retinoscopy was not possible. No intraocular inflammatory signs were observed.

On 23 May 2004, the right eye lens opacities reabsorbed. Mild posterior capsule opacity remains (fig 1D). VA without correction was 6/12 (20/40) and J10. Refraction disclosed $+1.25 \mathrm{D}$. The left eye VA and myopia remained unchanged.

Six weeks later sudden pain, redness, and loss of vision in the left eye occurred. A high IOP of $60 \mathrm{~mm} \mathrm{Hg}$, hazy cornea, mutton fat keratic precipitates with flare $3+$ and cells $4+$ were observed in the left eye anterior chamber. She was treated with corticosteroids and antiglaucoma drops. Two weeks later, a central tear of the posterior capsule with large cortical remnants within the capsular bag (fig 2A) and a multitude of floating lens remnants with a granulomatous inflammatory reaction were observed in the vitreous (fig 2B). Following complete arrest of the inflammatory processes and a return to normal of the IOP, medical treatment was discontinued 5 weeks after its initiation.

At her last visit on 21 November 2004, both eyes were quiet. Only mild scattered lens capsule opacities were detected in both eyes (figs $2 \mathrm{C}$ and 2D). The VA without correction was 6/12 (20/40) and J10 in both eyes. With correction $(+1.25)$ for distance and near addition $(+3.00)$, the VA in both eyes was 6/ $9(20 / 30)$ and $\mathrm{Jl}$ respectively. Multifocal glasses were prescribed.

\section{Comment}

A quiet and uneventful involution of the autologous lens occurred in the right eye when the child was 9 years old. The mechanism of this phenomenon is unclear and may be associated with abnormalities of the lens collagen and/or crystallines. The lens involution in the right eye was not associated with any noticeable symptom but for a drop in vision. Progressive clearing of the lens opacity was followed by emmetropisation of the initial refractive error and visual improvement in the left eye. Two years later, spontaneous involution of the lens in the other eye was associated with a marked intraocular granulomatous inflammatory reaction ("granulomatous uveitis") reminiscent of a phacoanaphylaxis reaction. This acute reaction was, most probably, associated with the "escape" of immune tolerance towards the autologous lens antigens.

We are not aware of previous reports in the literature describing similar ocular phenomena.

I Habil, E Cohen, I Karshai, D BenEzra Pediatric Ophthalmology Unit, Hadassah Hebrew University, Jerusalem, Israel

F Behar-Cohen

Rothschild Ophthalmic Foundation, Paris, France

D BenEzra, F Behar-Cohen U598, INSERM, Paris, France

Correspondence to: David BenEzra, MD, PhD, Hadassah Hebrew University Hospital, POB 12000 Jerusalem, Israel; benezra@md.huji.ac.il

doi: 10.1136/bjo.2005.076935

Accepted for publication 20 June 2005

\section{References}

1 Stickler GB, Belau PG, Farrell FJ, et al. Hereditary progressive arthro-ophthalmopathy. Mayo Clin Proc 1965;40:433-55.

2 Francomano CA, Liberfarb RM, Hirose T, et al. The Stickler syndrome is closely linked to COL2A1, the structural gene for type II collagen. Pathol Immunopathol Res 1988:7:104-6.

3 Donoso LA, Edwards AO, Frost AT, et al. Identification of a stop codon mutation in exon 2 of the collagen 2Al gene in large stickler syndrome family. Am J Ophthalmol 2002;134:720-7.

4 Spallone A. Stickler's syndrome: a study of 12 families. Br J Ophthalmol 1987;71:504-9.

\section{Temporal pterygium: benign or} not?

A true pterygium is a degenerative and hyperplastic process in which the cornea is invaded by a triangular fold of bulbar conjunctiva. Duke-Elder states that the pterygium when single is almost invariably found on the nasal side. ${ }^{1}$ The literature on pterygium is abundant and almost from the beginning the emphasis has been placed on its location on the nasal side.

Squamous cell neoplasia of the conjunctiva is relatively uncommon and can masquerade as common, but less significant, ocular surface conditions including pterygium or chronic blepharoconjunctivitis. We present a case of intraepithelial neoplasia, initially diagnosed as inflamed pterygium.

\section{Case report}

A 77 year old man, who had worked on the railways, presented with a 3 week history of redness on the outer aspect of the left eye. No history of associated pain, discharge, or watering was elicited.

His medical history included hypertension and hypercholesterolaemia under treatment.

Best corrected visual acuity in each eye was $6 / 5$. On inspection of the anterior segment, the left temporal conjunctiva showed a fleshy tissue encroaching on the temporal peripheral cornea (fig 1). The peripheral cornea showed an elevated ridge with punctate staining. The overlying conjunctiva was injected. The rest of the ocular examination was within normal limits.

A provisional diagnosis of inflamed pterygium of left eye was made and the patient was commenced on prednisolone $0.5 \%$ eye drops at this stage with advice to review in 2 weeks' time.

On follow up no significant change was noticed in the lesion. On further inquiry the patient gave a history of injury to left eye with hot ashes many years earlier. In view of

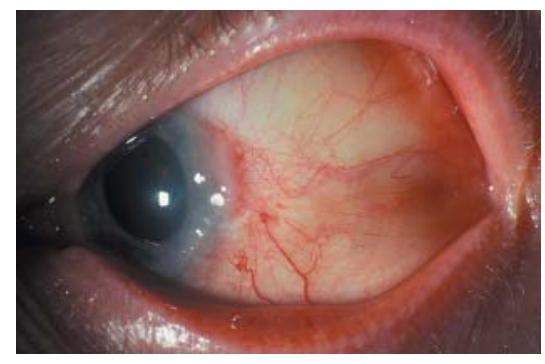

Figure 1 Left eye showing presence of a soft tissue lesion on the temporal conjunctiva encroaching on the limbus before local excision and radiotherapy.

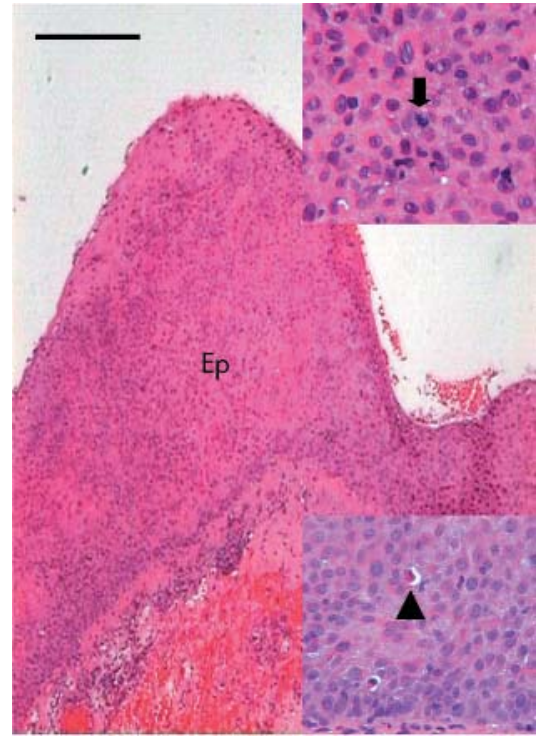

Figure 2 Section through the conjunctiva stained with haematoxylin and eosin. The main figure demonstrates grossly and irregularly thickened, dysplastic epithelium (Ep; scale bar, $100 \mu \mathrm{m})$. The inserts show an atypical mitosis (above, arrow) and dyskeratosis (below, arrowhead) within the epithelium.

the atypical location and the appearance of the lesion, we did an excision biopsy of the conjunctival and corneal lesion. Histopathology revealed an irregular epithelial thickening associated with dyskeratosis and full thickness dysplasia. Numerous mitotic figures, some atypical, were present throughout the epithelium (fig 2). A diagnosis of conjunctival intraepithelial neoplasia was made. Although no unequivocal evidence of invasion was seen in the multiple sections examined, fragmentation of the tissue during processing precluded confirmation of complete excision.

The patient was referred for further treatment to an ocular oncologist and underwent ruthenium plaque therapy followed by topical 5-fluorouracil treatment.

\section{Comment}

Temporal pterygium is reported, although Dolezalova found only one case of unilateral temporal pterygium out of 1388 Arab patients with pterygia. ${ }^{2}$ We would therefore consider this case to be atypical.

The role of pterygium in the development of ocular surface squamous neoplasia is unclear. $^{3}$ Both conditions have a strong association with exposure to ultraviolet-B radiation. Sevel and Sealy's study of 12 squamous cell carcinoma and 17 carcinoma in situ arising in 100 pterygia found that it can be difficult to distinguish a "reactive pterygium" from carcinoma in situ and malignant change should be considered in a pterygium if there is unusual evidence of invasion, extension, or if the lesion becomes particularly vascular. ${ }^{4}$

To our knowledge, the last reported case of temporal pterygium was in the 1970 s. $^{25}$ We present this case to refresh the memory and to highlight the importance of keeping an index of suspicion for squamous cell neoplasia in any atypical presentation of the more 
common conjunctival lesions such as pterygium.

B Ramasamy, S A Quah, M S Wishart

Department of Ophthalmology, North Cheshire Hospitals NHS Trust, Lovely Lane, Warrington WA5

1QG, UK

P Hiscott

Unit of Ophthalmology, Department of Medicine, University Clinical Departments, Duncan Building Daulby Street, Liverpool L69 3GA, UK

Correspondence to: Balasubramanian Ramasamy, Department of Ophthalmology, Warrington Hospita NHS Trust, Lovely Lane, Warrington WA5 IQG, UK anitharams@aol.com

doi: $10.1136 /$ bjo. 2005.071993

Accepted for publication 22 June 2005

Competing interests: none declared

\section{References}

1 Duke-Elder S. System of ophthalmology, Vol III, Part I. St Louis: Mosby, 1965:573-83.

2 Dolezalova V. Is the occurrence of a temporal pterygium really so rare? Ophthalmologica 1977; 174:88-91.

3 Lee GA, Hirst LW. Ocular surface squamous neoplasia. Surv Ophthalmol 1995;39:429-50.

4 Sevel D, Sealy R. Pterygia and carcinoma of the conjunctiva. Trans Ophthalmol Soc UK 1968:88:567-8

5 Awan KJ. The clinical significance of a single unilateral temporal pterygium. Can J Ophthalmol $1975 ; 10: 222-6$

\section{Simultaneous intraosseous and intradural capillary haemangioma of orbit}

Primary intraosseous haemangioma is an uncommon tumour of bone which tends to involve the vertebrae and skull. ${ }^{12}$ Bony orbital lesions are rare with very few case reports in the literature. ${ }^{134}$ Simultaneous intradural involvement has never been reported in association with an orbital component. We report an unusual case of capillary haemangioma of the orbital roof with periorbital and dural involvement.

\section{Case report}

A 39 year old white male was seen with a 1 year history of painless right upper eyelid swelling and reduced superior visual field. He had marked downward $(3 \mathrm{~mm})$, outward $(2 \mathrm{~mm})$, and axial $(4 \mathrm{~mm})$ displacement of the right globe (fig 1A), with limitation of elevation and 5 dioptres of hypotropia in upgaze. The remaining ocular and systemic evaluation were normal.

Contrast enhanced CT and gadolinium enhanced magnetic resonance imaging (MRI) (fig lB) demonstrated a well circumscribed faintly calcified mass centred within the bony roof of the right orbit. It was homogeneously isointense to grey matter on T1WI, slightly hyperintense on T2WI, and demonstrated marked homogeneous contrast enhancement. Transosseous extension intraorbitally was noted, with displacement of the superior rectus muscle, optic nerve, and globe inferiorly without evidence of invasion or encasement. Transosseous extension of the mass intracranially was completely extra-axial in location, with involvement of the adjacent dura. Provisional diagnosis in the absence of a known primary tumour, was intraosseous meningioma.

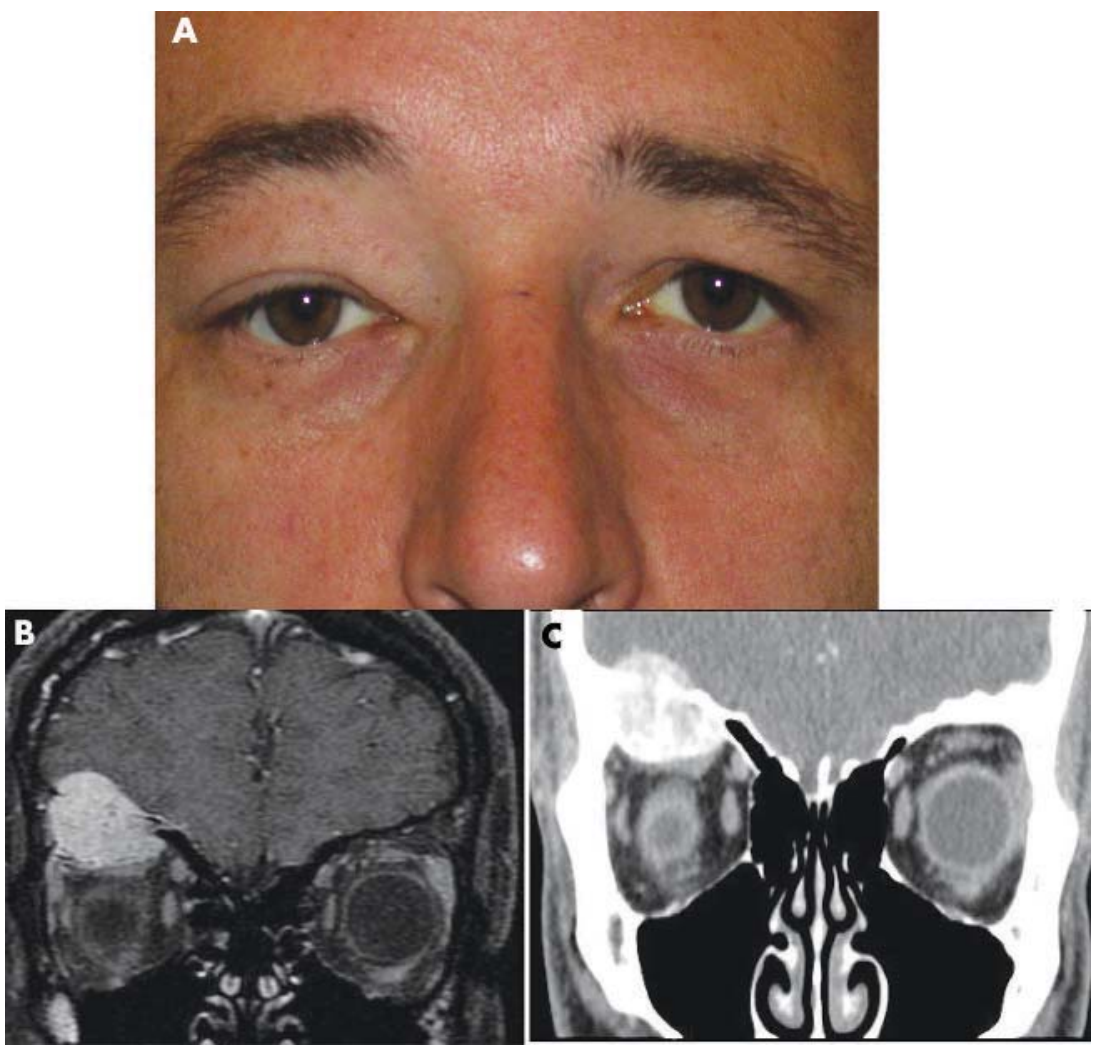

Figure 1 (A) A 39 year old patient showing proptosis and ptosis in the right eye. (B) Gadolinium enhanced coronal Ti fat saturated image through the orbits demonstrates an intraosseous mass in the right orbital roof, with intraorbital and intracranial extension. The intracranial portion was completely extra-axial, with associated dural involvement, as indicated by the thickened and enhancing dura adjacent to the dominant intracranial component. (C) Contrast enhanced coronal computed tomography (CT) image through the orbits demonstrates an intraosseous mass in the right orbital roof, with intraorbital and intracranial extension. Its heterogeneous appearance is the result, in part, of scattered calcifications throughout the mass. Mass effect upon the superior extraocular muscle group is evident.

The patient underwent right sided frontal craniotomy and orbital osteotomy with piecemeal gross total resection of the right orbital roof, the involved adjacent periorbita, dura and bone.

Grossly, pathological samples including dura (fig 2A) were soft and reddish-light tan coloured in appearance. Microscopic examination (fig 2B) revealed a cellular capillary haemangioma of bone, with periorbital and dural involvement (fig 2D), consisting of thin walled blood vessels with some osteoblastic activity and new bone formation. Tumour immunohistochemistry stains for CD34 (fig 2C), CD31, vimentin, and Ol3 were positive, confirming a vascular origin.

\section{Comment}

Intraosseous haemangiomas are benign tumours arising from the intrinsic blood vessels of bone and are two to three times more common in females than males. ${ }^{135}$ They are slow growing, accounting for only $0.7-1 \%$ of bone tumours, with the most common site being the vertebrae and skull (frontal and parietal). ${ }^{134}$ They are typically seen in the adult population, with a peak in the fourth decade, although any age can be affected. $^{13-6}$ Haemangiomas are histopathologically classified as either cavernous (common in the skull and orbit) or capillary (found mainly in vertebrae). ${ }^{1}$ The pathogenesis of these tumours is unknown.
The clinical presentation of orbital intraosseous haemangioma is usually a progressive asymptomatic mass which may lead to proptosis, diplopia, optic neuropathy, and ptosis. To date, the largest series ${ }^{3}$ contained 21 cases, of which four were of the capillary type. ${ }^{3}$ Though intracranial extension has been noted in the past, intradural lesion is reported only once with calvarial capillary haemangioma (sphenoid) $)^{5}$ but never with orbital invasion.

Plain films typically show bony erosion with scalloped bone giving a "sunburst" appearance. $^{145}$ Cavernous and capillary haemangiomas usually have similar imaging findings with differentiation made on histopathological analysis.

The differential diagnosis for a localised lytic bone lesion with calcifications is wide, including primary bone tumours such as osteosarcoma, chondrosarcoma, meningioma, haemangioma, brown tumour, or infection. Reactive lesions, such as xanthoma of bone, aneurysmal bone cyst, and reparative granuloma are also in the differential. Careful radiological evaluation in combination with clinical history and findings usually allows for differentiation among these different lesions.

With respect to our case, the characteristic high signal intensity on Tl imaging usually seen in vertebral haemangiomas was absent, probably the result of a relatively low fat content. ${ }^{12}$ 

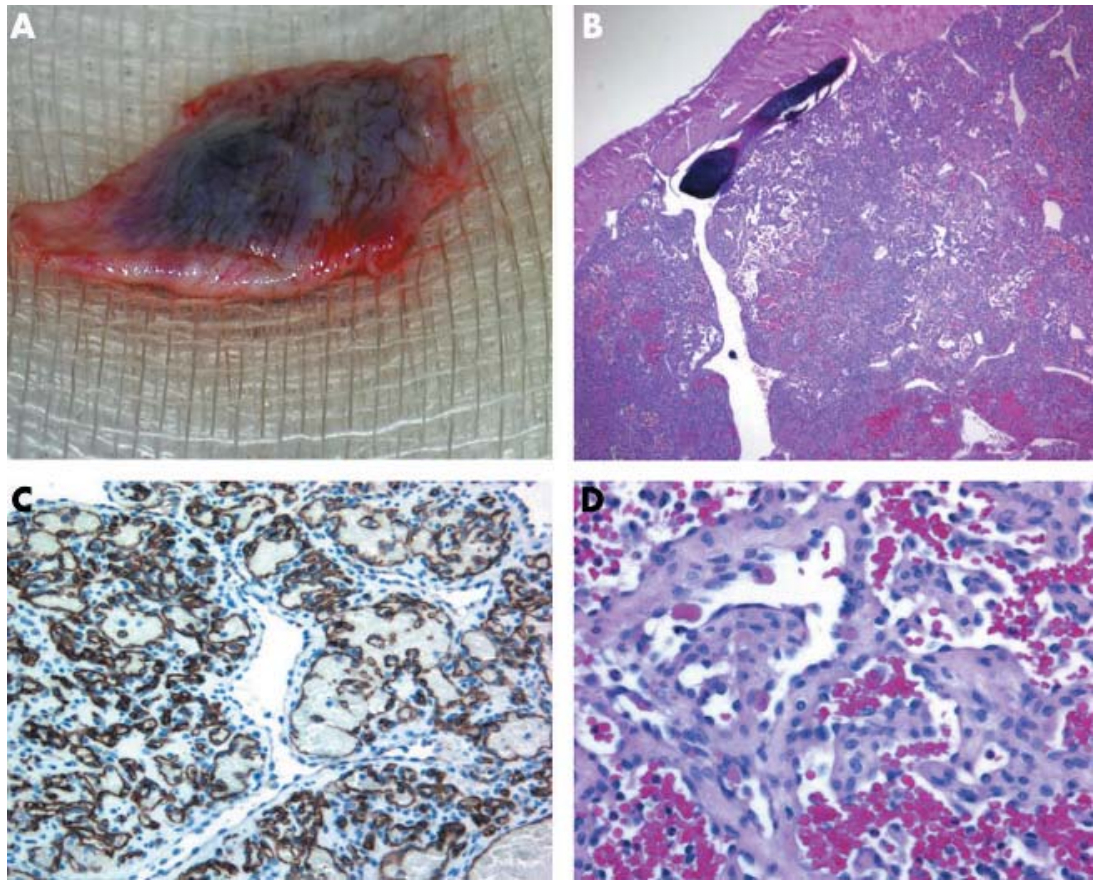

Figure 2 (A) Gross tumour mass showing involved resected dura. (B) HPE: $8 \times 4$ magnification showing thin walled blood vessels and osteoblastic activity of intraosseous cellular capillary haemangioma. (C) $6 \times 40$ magnification with CD 34 positivity confirming vascular origin. (D) $6 \times 40$ dural involvement by capillary haemangioma.

In our case, atypical dural enhancement on imaging was noted with associated erosion of overlying frontal bone.

Preferred treatment for symptomatic haemangiomas is surgical resection of the entire lesion, with preoperative embolisation. ${ }^{13-6}$ Radiation has been advocated for large and/ or unresectable lesions. ${ }^{147}$

N B Shah

Aravind Eye Hospital, Madurai, India

V A White

Department of Pathology, and Ophthalmology and Visual Sciences, VGH, UBC, Canada

$M$ Heran

Department of Radiology, VGH, UBC, Canada

C Haw

Department of Neurosurgery, VGH, UBC, Canada

J Rootman

Departments of Ophthalmology and Visual Sciences, and Pathology, VGH, UBC, Canada

Correspondence to: Dr Jack Rootman, Department of Ophthalmology and Visual Sciences, University of British Columbia, 2550 Willow Street, Vancouver, BC,

Canada V5Z 3N9; jrootman@interchange.ubc.ca

doi: 10.1136/bjo.2005.074427

Accepted for publication 6 June 2005

Competing interests: none declared

\section{References}

1 Sweet C, Richard S, Bharat Mehta. Primary intraosseous hemangioma of the orbit: CT and MR appearance. AJNR 1997;18:379-84.

2 Ross JS, Masaryk TJ, Modic MT, et al. Vertebral hemangioma: MR imaging. Radiology 1987; 165:165-9.
3 Relf SJ, Bartley GB, Unni KK. Primary orbital intraosseous hemangioma. Ophthalmology $1991 ; 98: 541-7$

Table 2 Two point LOD scores for linkage between the cataract locus and 13q markers in family B

\begin{tabular}{llllllll}
\multirow{2}{*}{$\begin{array}{l}\text { Marker } \\
\text { order }\end{array}$} & $\begin{array}{l}\text { Map } \\
\text { location }\end{array}$ & $\mathbf{0 . 0}$ & $\mathbf{0 . 1}$ & $\mathbf{0 . 2}$ & $\mathbf{0 . 3}$ & $\mathbf{0 . 4}$ & $\mathbf{0 . 5}$ \\
\hline D13S1316 & 0.00 & -1.13 & 1.34 & 1.08 & 0.66 & 0.25 & 0.00 \\
D13S1236 & 2.77 & 1.63 & 1.34 & 0.99 & 0.61 & 0.23 & 0.00 \\
D13S175 & 6.03 & 1.04 & 0.88 & 0.66 & 0.40 & 0.16 & 0.00 \\
D13S232 & 6.99 & -5.25 & -0.56 & -0.15 & 0.01 & 0.05 & 0.00 \\
D13S1243 & 9.79 & -6.19 & -0.76 & -0.32 & -0.12 & -0.03 & 0.00 \\
\hline
\end{tabular}

Pedigree and haplotype construction were undertaken using Cyrillic v.2.1 software (figs $1 \mathrm{~A}$ and $2 \mathrm{~A}$ ).

Table 1 Two point LOD scores for linkage between the cataract locus and Iq markers in family $\mathrm{A}$

\begin{tabular}{llllllll}
\hline \multirow{2}{*}{$\begin{array}{l}\text { Marker } \\
\text { order }\end{array}$} & $\begin{array}{l}\text { Map } \\
\text { location }\end{array}$ & $\mathbf{0 . 0 0}$ & $\mathbf{0 . 1 0}$ & $\mathbf{0 . 2 0}$ & $\mathbf{0 . 3 0}$ & $\mathbf{0 . 4 0}$ & $\mathbf{0 . 5 0}$ \\
\cline { 2 - 7 } D1S2651 & 142.24 & -4.49 & 0.23 & 0.25 & 0.14 & 0.01 & 0.00 \\
D1S2746 & 147.60 & -4.39 & 0.53 & 0.60 & 0.46 & 0.22 & 0.00 \\
D1S252 & 150.27 & -3.90 & 0.40 & 0.25 & 0.10 & 0.02 & 0.00 \\
D1S2344 & 153.59 & 1.44 & 1.29 & 1.03 & 0.70 & 0.32 & 0.00 \\
D1S442 & 154.74 & 0.43 & 0.04 & 0.25 & 0.10 & 0.02 & 0.00 \\
D1S498 & 155.89 & 2.40 & 1.95 & 1.46 & 0.94 & 0.42 & 0.00 \\
D1S2346 & 158.75 & 1.20 & 0.93 & 0.65 & 0.36 & 0.11 & 0.00 \\
D1S305 & 159.32 & 2.40 & 1.95 & 1.46 & 0.94 & 0.42 & 0.00 \\
D1S1595 & 161.05 & 0.49 & 0.50 & 0.41 & 0.25 & 0.08 & 0.00 \\
D1S2635 & 165.62 & 0.61 & 0.56 & 0.43 & 0.27 & 0.09 & 0.00 \\
D1S1167 & 168.52 & 2.44 & 1.95 & 1.46 & 0.94 & 0.43 & 0.00 \\
D1S2844 & 175.03 & 2.40 & 1.95 & 1.46 & 0.94 & 0.42 & 0.00 \\
D1S2878 & 177.86 & -4.75 & 0.09 & 0.23 & 0.22 & 0.14 & 0.00 \\
\hline
\end{tabular}
cataracts.

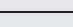

4 Hook SR, Font RL, Mc Cary JA, et al. Intraosseous capillary hemangioma of the frontal bone. Am J Ophthalmol 1987; 103:824-7.

Khanam H, Maurice HL, Charles LW, et al. Calvarial hemangiomas: report of 2 cases and review of the literature. Surg Neurol 2001;55:67-72

Hornblass A, Zaidman GW. Intraosseous orbital cavernous hemangior

7 Pandey SS, Pandey AK. Osseous hemangiomas. Arch Orthop Trauma Surg 1981;99:23-8.

Two novel mutations of connexin genes in Chinese families with autosomal dominant congenital nuclear cataract

onital or childhood cataract is a clinicolly and genetically highly heterogeneous (dince being most common. Non-syndromic congenital cataracts have an estiinthency of $1-6$ per 10000 live Underlying mutations have identified 14 genes involved in the pathogenesis of isolated inherited cataract, including seven genes for crystallins (CRYAA, CRYAB, CRYBAl/A3, CRYBB1, CRYBB2, CRYGC, membrane protein (LIM2 and MIP), one for beaded filament structural protein 2 (BFSP2), and one for glucosaminyl ( $\mathrm{N}$-acetyl) transferase 2 (GCNT2), one for heat shock transcription factor (HSF4). Here we report two novel heterozygous mutations in the GJA8 and GJA3 genes, in two Chinese families affected by autosomal dominant congenital nuclear 


\section{Case report}

We studied two Chinese three generation nuclear cataract families with a dominant pattern of inheritance. Clinical information and blood specimens were obtained from 16 members of family A (seven affected and nine unaffected), and 13 members of family B (nine affected and four unaffected). All participants had a full ocular assessment to document the phenotype. The phenotype of two families was characterised by bilateral nuclear cataract that was present at birth or developed during infancy. There was no evidence of other systemic or ocular defects.

After obtaining informed consent, we used a panel of 46 microsatellite markers to study 13 loci for known candidate genes of autosomal dominant congenital cataract susceptibility. The markers' order and position were obtained from the Marshfield Genetic Database (www.marshfield.org/genetics) maps). Genotyping and data collection were conducted by ABI Prism GeneMapper v 3.0 software. We carried out two point linkage analysis using the MLINK program from the Linkage v.5.10 software package. It suggested positive linkage on chromosome lq21.1 (lod score was 2.44 for marker D1S1167) in family A and chromosome 13q11-12 (lod score was 1.63 for marker D13S1326) in family B (tables 1 and 2).

There are two strong candidate genes in these regions, GJA8 encoding connexin $50(\mathrm{Cx} 50)$ and GJA3 encoding connexin 46 (Cx46). We screened the mutation of candidate genes by bidirectional sequencing polymerase chain reaction products $(300-$ $700 \mathrm{bp}$ ). Sequence analysis of the entire coding region and immediate flanking regions detected a heterozygous $191 \mathrm{~T} \rightarrow \mathrm{G}$ (AF217524) transition in exon 2 of GJA8, resulting in a Val $\rightarrow$ Gly substitution at codon 64 (fig 1B). Sequence analysis of GJA3 detected a heterozygous $134 \quad \mathrm{G} \rightarrow \mathrm{C}$ (AF075290) transition, resulting in a Trp(TGG) $\rightarrow$ Ser (TCG ) substitution at codon 45 (fig 2B). We examined all unaffected members of two families and 200 unrelated normal controls for GJA3 and GJA8 gene mutations but failed to detect these sequence variations.

\section{Comment}

Three connexins are expressed in the lens: connexin 43, connexin 46, and connexin 50 . Gap junction intercellular communication is an essential part of the cell-cell communication system, which facilitates the exchange of ions, metabolites, signalling molecules, and other molecules with a molecular weight up to $1 \mathrm{kDa}{ }^{2}$

Each gap junction channel is composed of two hemi-channels, or connexons, which dock in the extracellular space between adjacent cells, and each connexon comprised six integral transmembrane protein subunits known as connexins. All connexins have four transmembrane domains and two extracellular loops with cytoplasmic $\mathrm{N}$ and $\mathrm{C}$ termini.

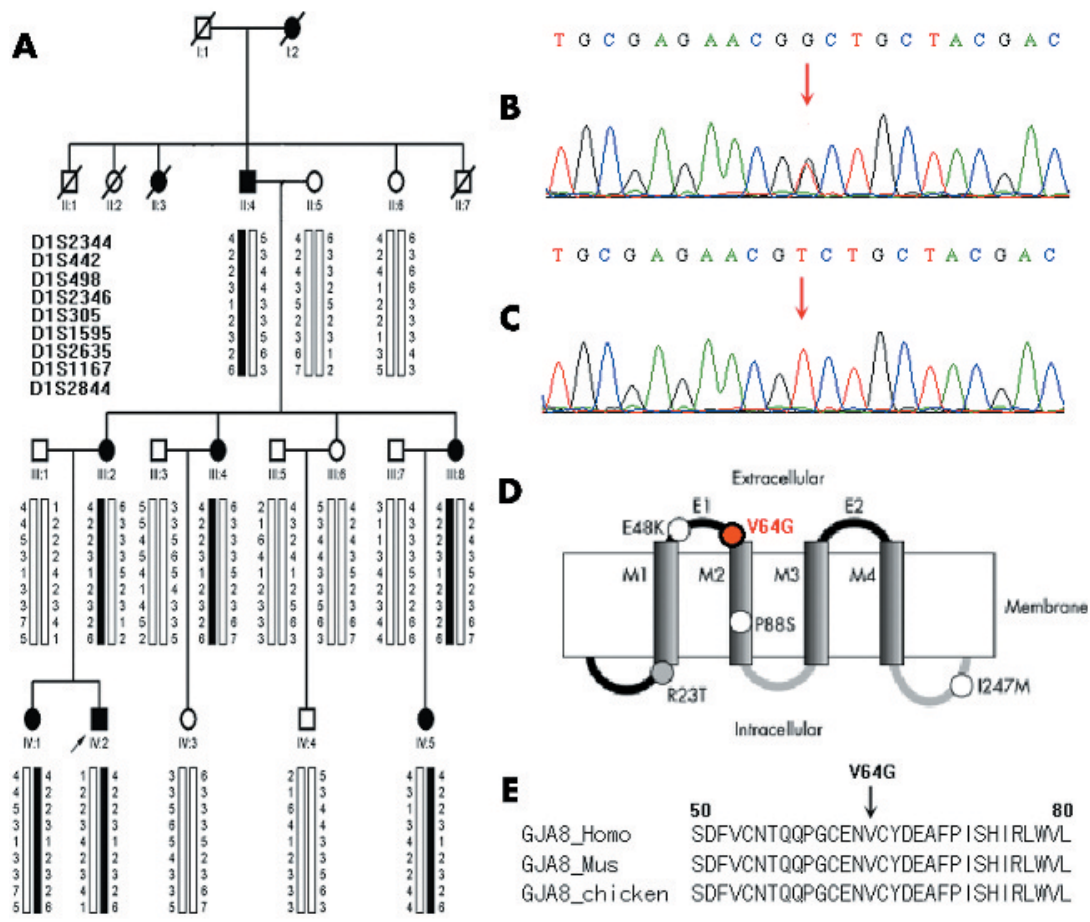

Figure 1 (A) Pedigree and haplotype analysis of family A showing segregating nine microsatellite markers on chromosome 1, listed in descending order from the centromere. Squares and circles symbolise males and females, respectively. Solid and open symbols denote affected and unaffected individuals, respectively. IV:2 is the proband. (B) Sequence chromatograms showing the heterozygous $191 \mathrm{~T} \rightarrow \mathrm{G}$ transition that converts a Val residue (GTC) to a Gly residue (GGC) at codon 64. (C) Sequence chromatograms of wild type allele. (D) Schematic diagram of the predicted $\mathrm{C} \times 50$ polypeptide and location of $\mathrm{V} 64 \mathrm{G}$ and known mutations. M1-M4, transmembrane domains $1-4$; E1 and E2, extracellular domains 1 and 2, respectively. (E) Cx50 multiple protein sequence alignment in different specices. Reference sequence numbers of protein are human (NP_005258), mouse (NP_032149), and chicken (NP_990328). The arrow directed the mutant amino acid residue.

To date, four heterozygous missense Cx50 mutations (P88S, E48K, R23T, and I247M) have been described, causing a nuclear or zonular nuclear pulverulent cataract. $^{3-6}$ Six mutations of Cx46 have been associated with ADCC, including five missense mutations (F32L, P59L, N63S, P187L, and N188T) and one insertion mutation (1137 insC), which resulted in a frame shift at codon 380 (S380fs)..$^{7-12}$

Currently, two mutations occurred: Cx50 (G22R and D47A) results in cataracts in the mouse, ${ }^{13} 14$ but no dominant spontaneous or mutagen induced cataracts have been associated with the murine gene for GJA3 (Gja3).

V64G and W45S substitutions in two Chinese families occurred within evolutionarily conserved residues across species for Cx50 and $\mathrm{Cx} 46$ (figs $1 \mathrm{E}$ and 2E). These two mutant amino acid residue locate at the phylogenetically conserved extracellular loop l (E1). The two extracellular loops mediate docking between connexons and the El loop has also been shown to be important for determinant of the transjunctional voltage required for closure of gap junction pores. ${ }^{15}$ The mutant proteins may disrupt normal interactions between the two connexons, which may reduce resistance of the intercellular channel to the leakage of small ions.

In conclusion, two novel heterozygous mutations, V64G in Cx50 and W45S in Cx46, were identified in two Chinese families. These further expand the genetic and phenotypic heterogeneity of cataract.

Z Ma, J Zheng, F Yang, J Ji, X Li, X Tang, $X$ Yuan, $X$ Zhang, $H$ Sun

Eye Center of Tianjin Medical University, Tianjin,

China

$\mathrm{Z} \mathrm{Ma}$

National Center of Human Genome Research (Beiijing), Beijing, China

Correspondence to: Huimin Sun, Eye Center of Tianiin Medical University, Tianjin, China; doctorsunhm@eyou.com

doi: 10.1136/bjo.2005.075184

Accepted for publication 1 June 2005

Competing interests: none declared

\section{References}

1 Lambert SL, Drack AV. Infantile cataracts. Surv Ophthalmol 1996;40:427-58.

2 White TW. Unique and redundant connexin contributions to lens development. Science 2002;295:319-20.

3 Shiels A, Mackay D, lonides A, et al. A missense mutation in the human connexin 50 gene (GJA8) underlies autosomal dominant "zonular pulverulent" cataract on chromosome lq Am J Hum Genet 1998;62:526-32.

4 Berry V, Mackay D, Khaliq S, et al. Connexin 50 mutation in a family with congenital "zonular nuclear" pulverulent cataract of Pakistani origin. Hum Genet 1999;105:168-70.

5 Polyakov AV, Shagina IA, Khlebnikova OV, et al. Mutation in the connexin 50 gene (GJA8) in a Russian family with zonular pulverulent cataract. Clin Genet 2001;60:476-8.

6 lloughby CE, Arab S, Gandhi R, et al. A novel GJA8 mutation in an Iranian family with progressive autosomal dominant congenital nuclear cataract. J Med Genet 2003;40:e124.

7 Mackay D, lonides A, Kibar Z, et al. Connexin46 mutations in autosomal dominant congenital cataract. Am J Hum Genet 1999:64:1357-64.

8 Gerido DA, White TW. Connexin disorders of the ear, skin, and lens. Biochim Biophys Acta 2004; 1662:159-70. 


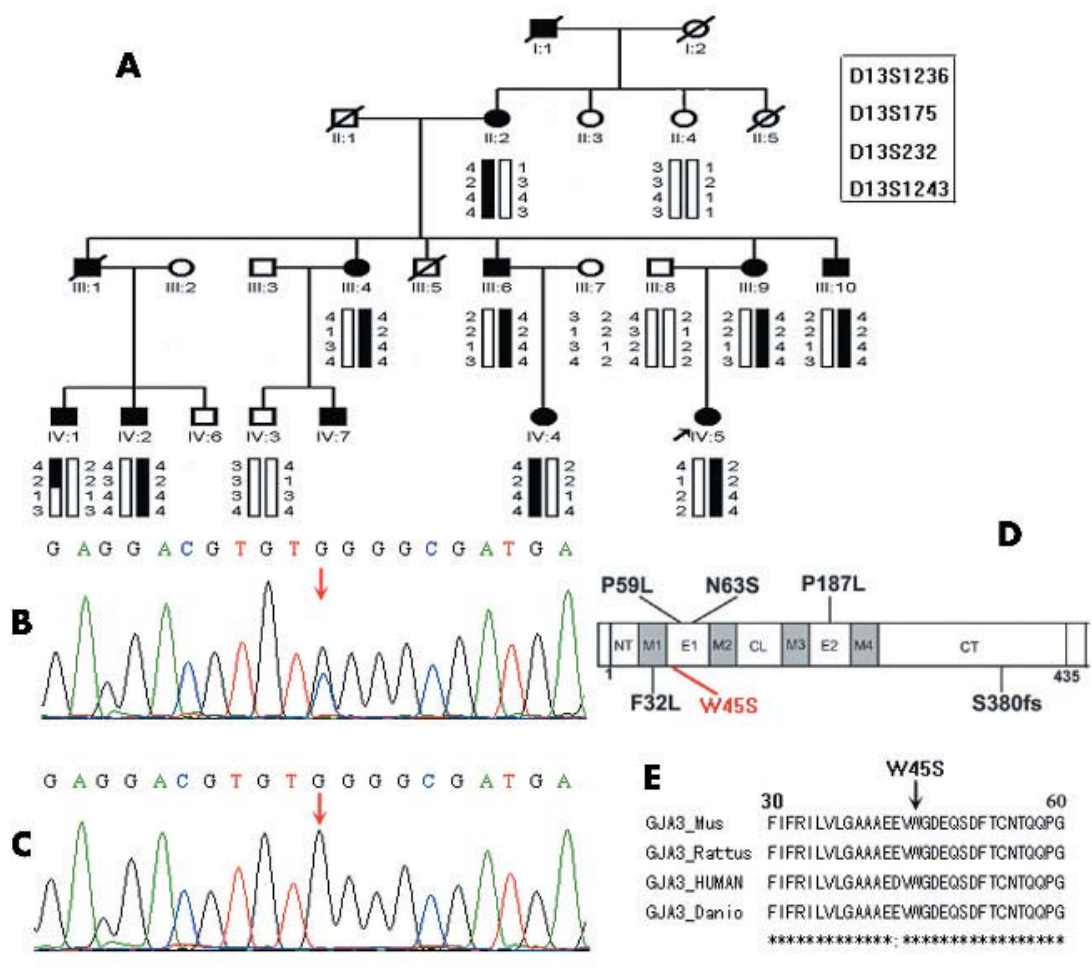

Figure 2 (A) Pedigree and haplotype analysis of family B showing segregation of four microsatellite markers on chromosome 13q. Squares and circles symbolise males and females, respectively. Solid and open symbols denote affected and unaffected individuals, respectively. IV:5 is the proband. (B) Sequence chromatograms showing the heterozygous $134 \mathrm{G} \rightarrow \mathrm{C}$ transition resulting in a Trp(TGG) $\rightarrow$ Ser (TCG ) substitution at codon 45 . (C) Sequence chromatograms of wild type allele. (D) Exon organisation and mutation profile of GJA3. Cx46 has nine structural domains including a cytoplasmic amino-terminus (NT), four transmembrane domains (M1-M4), two extracellular loops (E1-E2), a cytoplasmic loop (CL), and a cytoplasmic carboxy-terminus (CT). The relative locations of the W45S mutation and other mutations associated with dominant cataracts in humans are indicated. (E) Cx50 multiple protein sequence alignment in different species. Reference sequence numbers of protein are human (NP_068773), mouse (NP_058671), rat (Rattus norvegicus) (NP_077352), and zebrafish (Donio rerio) (NP_997525). The arrow directed the mutant amino acid residue.

9 Rees MI, Watts P, Fenton I, et al. Further evidence of autosomal dominant congenital zonular pulverulent cataracts linked to $13 \mathrm{q} 11$ (CZP3) and a novel mutation in connexin 46 (GJA3). Hum Genet 2000;106:206-9.

10 Jiang $\mathrm{H}$, Jin $\mathrm{Y}, \mathrm{Bu} \mathrm{L}$, et al. A novel mutation in GJA3 (connexin46) for autosomal dominant congenital nuclear pulverulent cataract. Mol Vis 2003;9:579-83.

11 Bennett TM, Mackay DS, Knopf HL, et al. A novel missense mutation in the gene for gapjunction protein alpha3 (GJA3) associated with autosomal dominant "nuclear punctate" cataracts linked to chromosome 13q. Mol Vis 2004; 10:376-82.

12 Li Y, Wang J, Dong B, et al. A novel connexin46 (GJA3) mutation in autosomal dominant congenital nuclear pulverulent cataract. Mol Vis 2004; 10:668-71.

13 Tusnady GE, Simon I. The HMMTOP transmembrane topology prediction server. Bioinformatics 2001;17:849-50.

14 Rong P, Wang X, Niesman I, et al. Disruption of Gja8 (a8 connexin) in mice leads to microphthalmia associated with retardation of lens growth and lens fiber maturation. Development 2002; 129:167-74.

15 Rubin JB, Verselis V, Bennett MVL, et al. domain substitution procedure and its use to analyze voltage dependence of homotypic gap junctions formed by connexins 26 and 32. Proc Natl Acad Sci USA 1992;89:3820-4.

\section{Pneumosinus dilatans in a 13 year old female}

Pneumosinus dilatans (PSD) is abnormal dilatation of paranasal sinuses that may occasionally present with visual symptoms. We present a case of PSD associated with sickle cell trait which occurred with visual deterioration.

\section{Case report}

A 13 year old female presented with gradual painless decrease of vision in both eyes for 1.5 years. Over this period her visual acuity dropped from 20/30 (RE) and 20/160 (LE) to hand motion in both eyes. Except for optic atrophy in both eyes, other ocular examinations were normal. In the visual field there was diffuse peripheral field loss and generalised depression. Past medical history was insignificant except for an appendectomy 5 years earlier.

An increased level of sickle cell haemoglobin which constituted $24.9 \%$ of her total haemoglobin was documented. $\mathrm{Her} \mathrm{Hb}_{\mathrm{A} 2}$ and $\mathrm{HbF}$ were in the normal range. She had anaemia with haemoglobin level of $9 \mathrm{~g} / \mathrm{dl}$, which we could not find any reason for.
Significant expansion of paranasal sinuses including maxillary, frontal, ethmoidal, and sphenoid sinus was visible on magnetic resonance images (MRI) of the patient as shown in figure 1 . Based on the MRI of the patient, the diagnosis of PSD would be appropriate.

Bilateral consecutive frontal craniotomy was performed in order to unroof the optic canal with the hope to release stretching of the optic nerve which we thought was the reason for her visual deterioration. Figure 1 (bottom) is an image of the surgical procedure. It is clear that the optic nerves have been entrapped in the bony canal and probably suffered from severe stretching and/or compressive effects. Six months after the procedure her visual acuity was 20/1200 in both eyes.

\section{Comment}

Pneumosinus dilatans is an abnormal dilatation of one or more of the paranasal sinuses. It has diverse manifestations including progressive visual loss if the sphenoid sinus is involved and/or if it is associated with optic nerve meningioma. If the ethmoidal sinus is involved it may present with proptosis. ${ }^{1}$ Although a valve mechanism raising the pressure inside the sinus is thought to be responsible for this condition, ${ }^{2}$ the exact etiology is unknown. ${ }^{3}$ In case of optic nerve damage the nerve is usually compressed in long bony tubes. ${ }^{4}$ Pneumosinus dilatans has been associated with meningioma of the intracanalicular optic nerve and anterior chiasmal angle, ${ }^{56}$ middle cranial fossa arachnoid cyst, ${ }^{7}$ cerebral hemiatrophy, and prolonged cerebrospinal fluid shunting. ${ }^{8}$

To our knowledge this is the first case of PSD associated with sickle cell trait. PSD has not been associated previously with haematological disorders. Considering the fact that sickle cell trait is generally an asymptomatic condition and the patient's mother was also an asymptomatic carrier, an aetiological relation is unproved. On the other hand, both conditions are rare in our population, therefore the probability of coincidence by chance would seem to be extremely low. The question remains whether our patient had an unusual form of sickle cell trait associated with gross bony involvement and deformity.

Different treatments have been proposed for PSD. These include subtotal resection of the medial wall of the maxillary sinus by an endoscopic approach, ${ }^{9}$ osteotomy of the deformed fronto-orbital bossing, and obliteration of the sinus with fat. ${ }^{10}$ Because of global and massive expansion of the sinuses and severe optic nerve dysfunction in this case, we preferred to decompress the optic nerve by removing the roof of bony canal which surrounded the intracranial optic nerve. This resulted in mild visual improvement.

M S Sanjari, M Modarreszadeh, K Tarassoly Ophthalmology Department Eye Research Center, Rasool Akram Hospital, Tehran, Iran

Correspondence to: Kia Tarassoly, MD, Ophthalmology Department, Eye Research Center, Rasool Akram Hospital, Tehran, Iran; kiatarassoly@ hotmail.com

doi: 10.1136/bjo.2005.069567

Accepted for publication 1 March 2005 

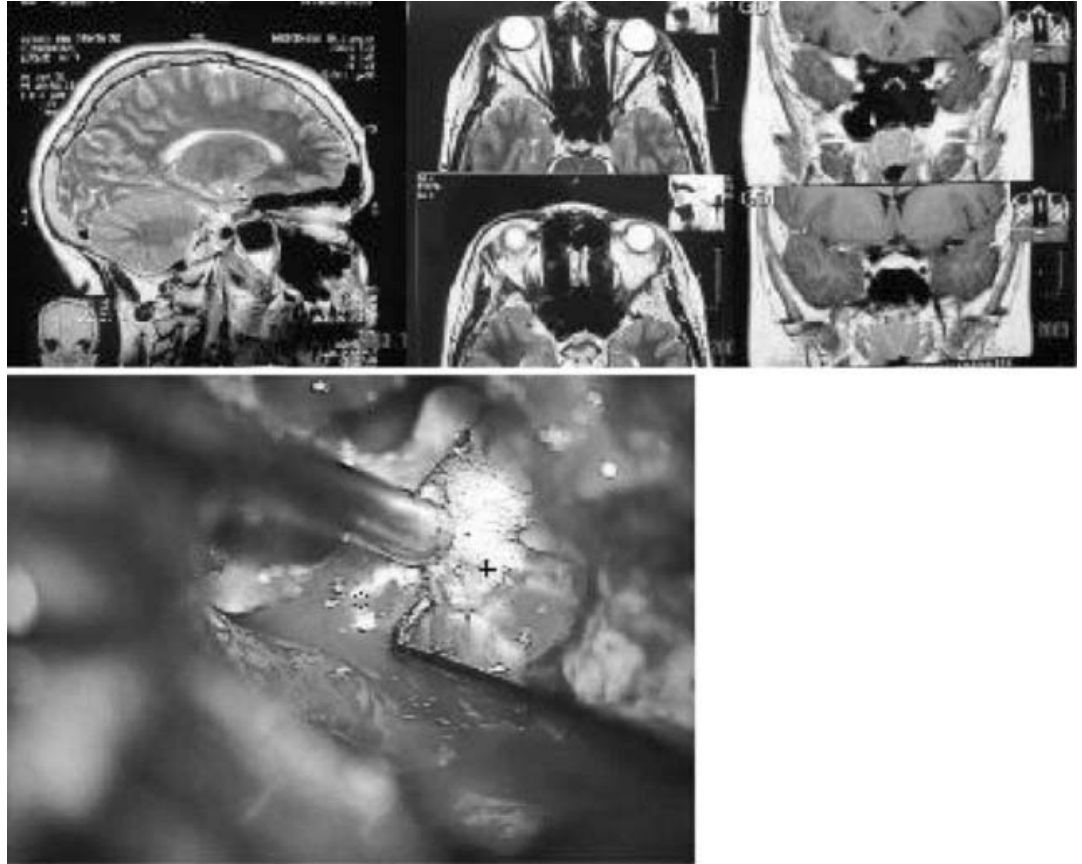

Figure 1 (Top) Magnetic resonance images show significant dilatation of paranasal sinuses. (Bottom) Surgical field image. "O" is intracranial part of optic nerve, " + " is the bony canal after partial removal; the metal instrument is a suction device tip.

\section{References}

1 Skolnick CA, Mafee MF, Goodwin JA Pneumosinus dilatans of the sphenoid sinus presenting with visual loss. I Neuro-ophthalmol 2000;20:259-63

2 Wolfensberger $M$. Pathogenesis of pneumosinus maxillaris dilatans. HNO 1984;32:518-20.

3 Walker JL, Jones NS. Pneumosinus dilatans of the frontal sinuses: two cases and a discussion of its aetiology. J Laryngol Otol 2002;116:382-5.

4 Stretch JR, Poole MD. Pneumosinus dilatans as the aetiology of progressive bilateral blindness. Br J Plast Surg 1992:45:469-73.

5 Hirst LW, Miller NR, Hodges FJ 3rd, et al. phenoid pneumosinus dilatans. A sign of meningioma originating in the optic canal. Neuroradiology 1982;22:207-10.

6 Mai A, Karis J, Sivakumar K. Meningioma with pneumosinus dilatans. Neurology 2003;60:1861

7 Redla S, Husami Y, Colquhoun IR. Apparent paradoxical vault changes with middle cranial fossa arachnoid cysts - implication for aetiology. Clin Radiol 2001;56:851-5.

8 Van Schayck R, Niedeggen A. Pneumosinus dilatans after prolonged cerebrospinal fluid shunting in young adults with cerebral hemiatrophy. A report of two cases and review of the literature. Neurosurg Rev 1992;15:217-23.

9 Juhl HJ, Buchwald C, Bollinger B. An extensive maxillary pneumosinus dilatans. Rhinology 2001:39:236-8.

10 Tellado MG, Mendez R, Lopez-Cedrun JL, et al. Pneumosinus dilatans of the frontal and ethmoidal sinuses: case report. J Craniomaxillofac Surg 2002;30:62-4.

Pellucid marginal degeneration coexistent with cornea plana in one member of a family exhibiting a novel KERA mutation

Characterised by flattening of the normally convex corneal surface, small corneas, high hyperopia, and arcus senilis, autosomal recessive cornea plana is secondary to KERA mutation. ${ }^{1-3}$ KERA encodes keratocan, an

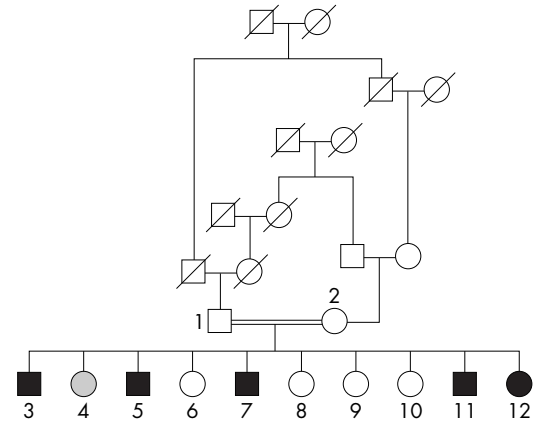

Cornea plana

Pellucid marginal degeneration and cornea plana

Figure 1 The family pedigree.

the rule" astigmatism, and absence of opacity. ${ }^{4}$ We report a case of superior PMD coexistent with cornea plana in a family exhibiting a novel KERA mutation and document the ophthalmic findings of the family.

\section{Case series}

Twelve individuals from a Saudi nuclear family were studied after institutional review board approval and family informed consent had been obtained from the family. Clinical findings and diagnoses are summarised in figures 1 and 2, and table 1 . Only one family member (patient 4 ) had a history of progressive visual difficulty over the last several years, and this was due to an increasing astigmatic refractive error. Axial lengths and keratometry readings were recorded using the Zeiss IOL-Master (2001 model), and corneal topography was performed using the Bausch \& Lomb Orbiscan 2Z (2002 model).

All family members underwent KERA DNA sequencing using methods previously described. ${ }^{3}$ A novel mutation was detected
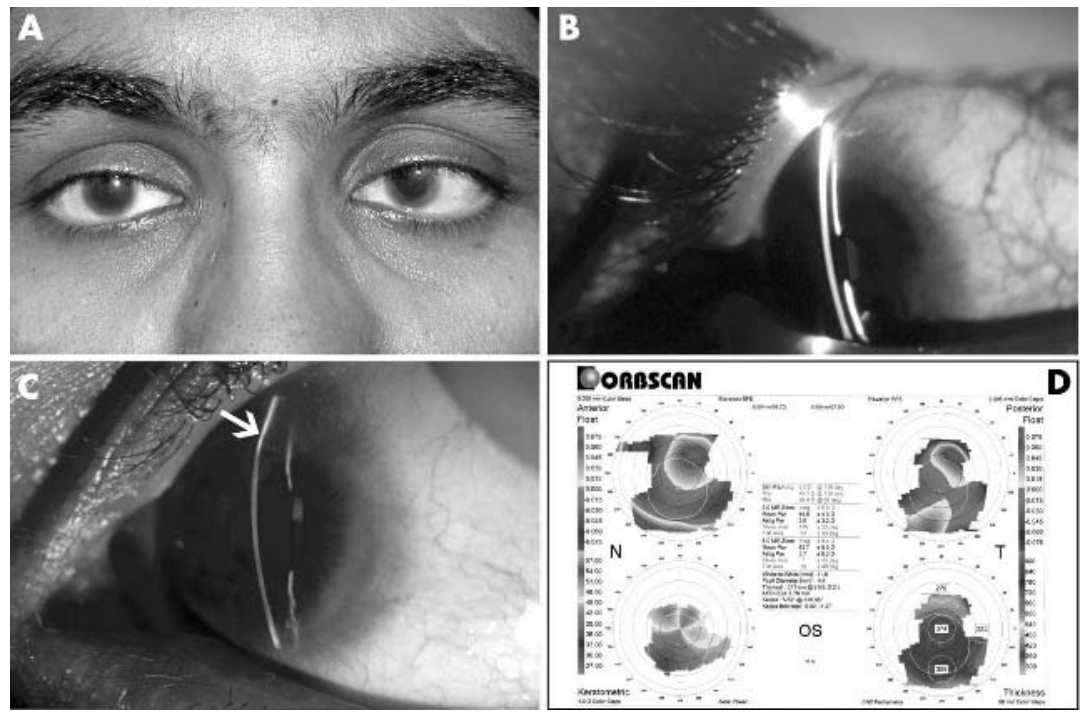

Figure 2 (A) The small flat corneas of a typical patient (No 3) are shown. (B) The slit lamp appearance of patient 3 is shown. (C) In addition to small flat corneas and early arcus senilis, patient 4 also demonstrated superior corneal thinning (arrow, LE) with associated corneal ectasia characteristic of superior pellucid marginal corneal degeneration. (D) Topography, LE of patient 4 shows the characteristic high astigmatism of superior pellucid marginal corneal degeneration. 


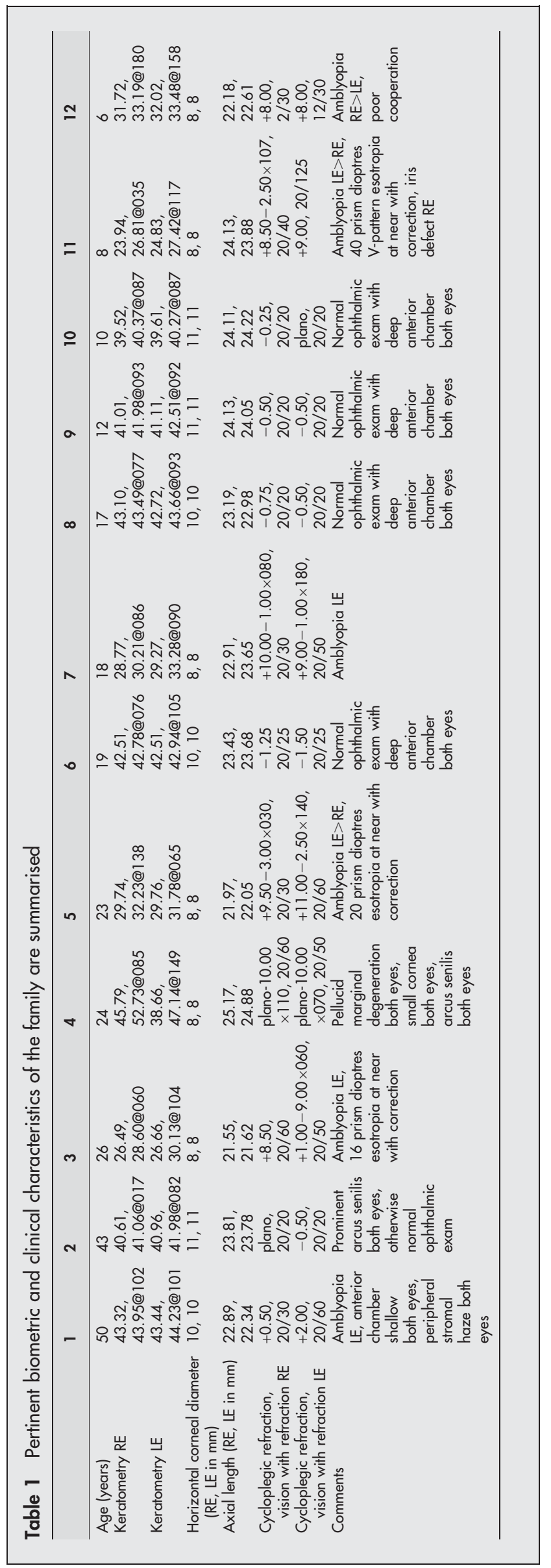

in exon 2, [1454 C>T, ENST00000266719], changing an arginine amino acid at position 279 to a stop codon [R279X]. The resultant truncated protein lacks the terminal 73 amino acids of normal keratocan. This mutation was homozygous in the five siblings with clinically evident cornea plana (Nos 3, 5, 7, $11,12)$ and the sister with clinical findings of PMD and cornea plana (No 4). All other family members (Nos 1, 2, 6, 8, 9, 10) were heterozygous for the mutation and clinically unaffected.

\section{Comment}

All four previously reported KERA mutations disrupt keratocan LRR architecture and are associated with similar corneal phenotypes in documented homozygotes. ${ }^{1-3}$ The current mutation [R279X] similarly disrupts LLR function, as the prematurely truncated protein lacks two LRRs of normal keratocan and is associated with the expected cornea plana phenotype. Interestingly, one homozygous individual (No 4) demonstrates corneal findings compatible with both superior PMD (corneal thinning with astigmatism) and autosomal recessive cornea plana (small corneas, arcus senilis ) - the presence of arcus senilis excludes classic PMD alone by definition. ${ }^{45}$ It is unlikely that the KERA mutation itself is responsible for the PMD findings in this individual. The sectorial thinning and progressive high astigmatism characteristic of superior PMD have not been reported in individuals documented to be homozygous for KERA mutation or in other pedigrees consistent with autosomal recessive cornea plana. ${ }^{1-36}$ The PMD findings of patient 4 are most likely the result of coincidence-that is, the occurrence of both cornea plana and PMD in the same individual. However, a defect in a poorly understood mechanism other than KERA itself that is responsible for normal keratocan function cannot be completely excluded as an explanation for these findings. ${ }^{7}$

A O Khan

Division of Pediatric Ophthalmology, King Khaled Eye Specialist Hospital, PO Box 7191, Riyadh 11462, Saudi Arabia

M Aldahmesh, A Al-Saif, B Meyer Aragene Project, King Faisal Specialist Hospital and Research Center, MBC 03-8, PO Box 3354, Riyadh 11211, Saudi Arabia

Correspondence to: Arif $O$ Khan, MD, Division of Pediatric Ophthalmology, King Khaled Eye Specialist Hospital, PO Box 7191, Riyadh 11462, Saudi Arabia; arif.khan@mssm.edu

doi: 10.1136/bjo.2005.073510

Accepted for publication 1 May 2005

\section{References}

1 Pellegata NS, Dieguez-Lucena JL, Joensuu T, et al. Mutations in KERA, encoding keratocan, cause cornea plana. Nat Genet 2000;25:91-5.

2 Lehmann OJ, El-Ashry MF, Ebenezer N, et al. A novel keratocan mutation causing autosomal recessive cornea plana. Invest Ophthalmol Vis Sci 2001;42:3118-22.

3 Khan AO, Al-Saif A, Kamburosis M. A novel KERA mutation associated with autosomal recessive cornea plana. Ophthal Genet 2004;25: 147-52.

4 Sridhar MS, Mashesh S, Bansal AK, et al. Pellucid marginal corneal degeneration. Ophthalmology 2004;111:1102-7. 
5 Sridhar MS, Mahesh S, Bansak AK, et al. Superior pellucid marginal corneal degeneration. Eye 2004; 18:393-9.

6 Forsius $\mathbf{H}$, Damsten $M$, Eriksson AW, et al. Autosmal recessive cornea plana. A clinical and genetic study of 78 cases in Finland. Acta Ophthalmol Scand 1998;76:196-203.

7 Dipple KM, McCabe ERB. Phenotypes of patients with "simple" Mendelian disorders are complex traits: thresholds, modifiers, and systems dynamics. Am J Hum Genet 2000;66:1729-35.

\section{Alteration of cyclic frequency by botulinum toxin injection in adult onset cyclic esotropia}

Cyclic strabismus is an uncommon disorder in which strabismus comes and goes alternately, consistently, and repetitively over a period of time. In a 48 hour cycle, a 24 hour period of orthotropia would be followed by a 24 hour period of constant strabismus. Cycles of 24 hour $^{1}$ to 96 hour $^{2}$ patterns have been reported. Most cases have been described in children, and the aetiology of cyclic strabismus is still speculative.

\section{Case report}

A 57 year old woman was referred to Kaohsiung Medical University Hospital with the complaint of a periodic visual fluctuation of a "good day" and a "bad day" alternately for about 6 months. She had diplopia on bad days. She did not have diabetes or hypertension. There was no history of strabismus, amblyopia, patching therapy, ocular trauma, or oculomotor palsy. She had received trials of Mestinon treatment by two neurologists. Except for pterygium excision 4 years earlier, other ocular and medical history were unremarkable. There was no family history of strabismus.

Her visual acuity was $20 / 25$ with +1.25 lens RE and 20/20 LE plano. Cycloplegic refraction was $+1.25 \mathrm{RE}$ and +0.50 LE. The anterior segments were normal except for recurrent pterygia on the nasal limbus in both eyes.

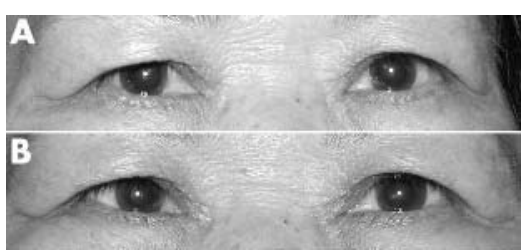

Figure 1 (A) "Good day"-orthotropia; (B) "Bad day" - esotropia.

Ophthalmoscopic examination, ocular alignment (fig 1A), and ocular motility were normal. Since the initial examination was on her "good day," she was asked to come back the next day-that is, on the "bad day."

The next day, there was a 25 prism dioptre, commitant right esotropia (fig 1B) with full ocular motility. The visual acuity was unchanged. Brain and orbit magnetic resonance imaging studies were unremarkable except for a suspected small arachnoid cyst on the right side of the falx.

She received $2.5 \mathrm{U}$ botulinum toxin (Botox) injection in her right medial rectus muscle (MR). The alignment was orthotropia 1 week after the injection. She was asymptomatic for about 2 months, but the cyclic pattern returned with a 96 hour cycle by patient history. A repeated $2.5 \mathrm{U}$ Botox injection in right $\mathrm{MR}$, which was given 3 months after the first, produced another asymptomatic period of 2 months. Two months after the second injection, she experienced constant strabismus without cyclic pattern, which persisted for about 1 year. She received right MR recession by $4 \mathrm{~mm}$ and right lateral rectus muscle resection by $5 \mathrm{~mm}$ for constant esotropia of 25 prism dioptres. After the surgery, the alignment was orthotropic and no recurrent of the cyclic pattern during 1 month of follow up. The stereopsis was 200 seconds of arc by Titmus test.

\section{Comment}

Adult onset cyclic strabismus is rare, ${ }^{2-8}$ and, to the best of our knowledge, only 10 patients have been reported. The reported cases of adult onset cyclic strabismus are summarised in table 1 . The patients had various ages of onset between 21 and 67 years. Most reported cases demonstrated 48 hour cyclic patterns. The persistence of the cycles, if not interrupted by surgery, was as long as 7 years. ${ }^{8}$ It is interesting that adult onset cyclic strabismus occurs predominantly in females and is frequently related to ocular or orbital diseases, trauma, or surgery. ${ }^{4-8}$

Botox injection has been used as treatment of cyclic strabismus. ${ }^{7}$ However, no change of the cyclic pattern was mentioned. We noted that the cyclic pattern in our patient changed 3 months after the first Botox injection, and the cycles were eliminated 2 months after the second injection.

The characteristics of cyclic strabismus in children are an average age of onset between 3 and 4 years, moderate hyperopia, and moderate angle. ${ }^{3}$ However, a female preponderance was not noted in childhood onset cyclic esotropia. No pertinent explanation for cyclic strabismus has been reported. Although Botox only has a temporary effect, both Botox injection ${ }^{7}$ and eye muscle surgery ${ }^{3}$ produce good ocular alignment results. More evidence and further investigation are required to elucidate the mystery.

\section{Acknowledgements}

The authors thank Professor William F Hoyt and Professor Creig S Hoyt for their review and criticism of this letter.

Y-H Lai

Department of Ophthalmology, Kaohsiung Medical University Hsiao-Kang Hospital, Kaohsiung Medical University, Kaohsiung, Taiwan

D R Fredrick

Department of Ophthalmology, University of California, San Francisco, San Francisco, CA, USA

Table 1 Summary of the adult onset cyclic strabismus

\begin{tabular}{|c|c|c|c|c|c|c|c|c|}
\hline $\begin{array}{l}\text { Case } \\
\text { No }\end{array}$ & $\begin{array}{l}\text { Age of } \\
\text { onset } \\
\text { (years) }\end{array}$ & Sex & $\begin{array}{l}\text { Cyclic } \\
\text { pattern }\end{array}$ & $\begin{array}{l}\text { Duration } \\
\text { of } \\
\text { cycles }\end{array}$ & Angle ( $\Delta)$ & $\begin{array}{l}\text { Related diseases } \\
\text { or coexistent conditions }\end{array}$ & Outcome & References \\
\hline 1 & 34 & Male & 4 days & 3 years & ET 35 & $\begin{array}{l}\text { Optic atrophy both eyes, alcohol } \\
\text { abuse }\end{array}$ & No treatment & Frenkel $^{2}$ \\
\hline 2 & 32 & Female $^{\star}$ & 2 days & NA & ET 35 & NA & No treatment, CPP? & Helveston ${ }^{3}$ \\
\hline 3 & 53 & Female & 2 days & NA & $\begin{array}{l}\text { XT 15, } \\
\text { RHT } 30\end{array}$ & Graves' disease & OT after muscle surgery & Knapp ${ }^{4}$ \\
\hline 4 & 55 & Female & 2 days & NA & RHT 25 & Graves' disease & OT after muscle surgery & Knapp $^{4}$ \\
\hline 5 & 67 & Female & 2 days & 5 years & $\begin{array}{l}\text { ET 25, } \\
\text { RHT } 8\end{array}$ & $\begin{array}{l}\mathrm{RD} R E, 360^{\circ} \text { encircling scleral } \\
\text { buckling procedure RE, cyclic } \\
\text { mydriasis and ptosis }\end{array}$ & No treatment, CPP & Troost $^{5}$ \\
\hline 6 & 34 & Female & 2 days & 9 weeks & $\begin{array}{l}\text { LHT } 20 \text {, } \\
\text { XT } 10\end{array}$ & $\begin{array}{l}\text { Craniofacial surgery for fronto- } \\
\text { orbital fibrous dysplasia, left side }\end{array}$ & OT after muscle surgery & Metz $^{6}$ \\
\hline 7 & 46 & Female & 2 days & 1 year & $\begin{array}{l}\text { ET } 12 \text { to } \\
45\end{array}$ & ECCE RE, high myopia RE & $\begin{array}{l}\text { Botulinum toxin injection, ET } \\
2 \Delta \text { with cycle eliminated after } \\
\text { muscle surgery }\end{array}$ & Riordan-Eva ${ }^{7}$ \\
\hline 8 & 21 & Female & 5 days? & 2 years & ET 25 & $\begin{array}{l}\text { RD RE, vitreolensectomy and } \\
\text { silicone oil exchange RE } \\
\text { ECCE RE, RD and PVR RE, PVT }\end{array}$ & Botulinum toxin injection, CPP & Riordan-Eva ${ }^{7}$ \\
\hline 9 & 49 & Female & 2 days & 7 years & $\begin{array}{l}\text { RHT } 15 \text {, } \\
\text { ET } 25\end{array}$ & $\begin{array}{l}\text { and scleral buckling RE, ECCE LE, } \\
\text { prophylactic encircling band } \\
\text { surgery LE }\end{array}$ & OT after muscle surgery & Bagheri $^{8}$ \\
\hline 10 & 57 & Female & 2 days & lyear & ET 30 & Recurrent pterygia in both eyes, & $\begin{array}{l}\text { Botulinum toxin injection, } \\
\text { cyclic pattern changed, } \\
\text { OT after muscle surgery }\end{array}$ & Present report \\
\hline
\end{tabular}

*Information provided by Dr Eugene Helveston (personal communication). NA, not available; CPP, cyclic pattern persisted; $\Delta$, prism dioptre; ET, esotropia; XT, exotropia; RHT, right hypertropia; LHT, left hypertropia; OT, orthotropia; RD, retinal detachment; PVR, proliferative vitreoretinopathy; ECCE, extracapsular cataract extraction; PVT, posterior vitrectomy. 
Correspondence to: Yu-Hung Lai, Department of Ophthalmology, Kaohsiung Medical University HsiaoKang Hospital, Kaohsiung Medical University, 100 Zihyou 1st Road, Kaohsiung, 807, Taiwan; yuhung lai@msa.hinet.net

\section{doi: $10.1136 /$ bjo.2005.069021}

Accepted for publication 3 May 2005

\section{References}

1 Windsor CE, Berg EF. Circadian heterotropia. Am J Ophthalmol 1969:67:565-71.

2 Frenkel RE, Brodsky MC, Spoor TC. Adult-onset cyclic esotropia and optic atrophy. J Clin Neuroophthalmol 1986;6:27-30.

3 Helveston EM. Cyclic strabismus. Am Orthopt J 1973;23:48-51

4 Knapp P. Special types of muscle anomalies associated with Graves' disease. Ophthalmology 1979;86:2081-4.

5 Troost BT, Abel L, Noreika J, et al. Acquired cyclic esotropia in an adult. Am J Ophthalmol 1981;91:8-13.

6 Metz HS, Searl SS. Cyclic vertical deviation. Trans Am Ophthalmol Soc 1984;82:158-65.

7 Riordan-Eva P, Vickers SF, McCarry B, et al. Cyclic strabismus without binocular function. $\checkmark$ Pediatr Ophthalmol Strabismus 1993;30:106-8.

8 Bagheri A, Ahmadieh H, Repka MX. Acquired cyclic strabismus in an adult. J Pediatr Ophthalmol Strabismus 2002;39:310-12.

\section{Hand hygiene in routine glaucoma clinics}

Nosocomial infection occurs via the hands of healthcare workers (HCWs). ${ }^{1}$ Hand hygiene reduces hospital infection rates; however, HCWs seldom comply with this. ${ }^{2}$

We determined how often ophthalmologists and allied professionals cleaned their hands and whether intervention was effective.

\section{Participants, methods, and results}

We conducted the study in the daily glaucoma clinics of Moorfields Eye Hospital where policy states that all HCWs must clean their hands between patients.

For 1 week, hand hygiene practice was monitored covertly by two observers. Potential hand cleaning opportunities were before or during patient contact, before or after manipulative procedures, and after glove removal. Manipulative procedures were defined as 5-fluorouracil subconjunctival injection, taking an eye swab, suture, or supramid removal, and bleb needling or massage.

Without revealing how the study was conducted, preliminary results were presented and also distributed by memo. Two weeks after this intervention, hand hygiene was re-monitored for 1 week.

Baseline hand hygiene episodes were 18\% but increased significantly to $28 \%(\mathrm{p}=0.005)$ following intervention (table 1). Before intervention two out of seven people performing procedures cleaned their hands, but not for the single episode that 5 -fluorouracil was used. However, after intervention six out of seven HCWs cleaned their hands $(p=0.04)$, including all three episodes in which 5fluorouracil was handled.

Before intervention, female HCWs cleaned their hands significantly more than males $(30 \% \vee 9 \%, \mathrm{p}<0.001)$. After intervention hand hygiene increased further for females $(54 \%, \mathrm{p}<0.001)$ with no change for males $(11 \%, \mathrm{p}=0.57)$.

Nurses had the highest frequency of hand cleaning but with no change after intervention $(69 \% \vee 58 \%, p=0.36)$. Increased hand hygiene was significant for doctors following intervention $(11 \% \vee 20 \%, \mathrm{p}=0.01)$.

\section{Comment}

Recently, nosocomial infection has attracted considerable media interest. While problematic worldwide, the United Kingdom has one of the highest rates of methicillin resistant Staphylococcus aureus (MRSA). ${ }^{3}$ The hands of HCWs are a major route of transmission. Hand hygiene frequencies range from $3 \%,{ }^{2}$ increasing to more than $60 \%$ when HCWs are aware of being observed. ${ }^{4}$

In our study, hand hygiene was low (18\%). Although significant improvement followed intervention $(28 \%)$ this was far from the hospital standard. Our new level of hand cleaning is likely to be transient as all but one study has demonstrated sustained improvement.
Previous studies, including our own, have shown that female HCWs clean their hands more often than males. ${ }^{6}$ In general, sex differences in hand washing are explained by the social role theory-that is, females are communal whereas men are agentic. ${ }^{7}$ Hence, women are more likely than men to participate in socially acceptable behaviour such as hand washing. ${ }^{8}$ In our study, intervention produced a significant improvement in hand hygiene for females with no effect on males. Behaviourally, men are less easily influenced than women, ${ }^{7}$ which may explain why intervention had no effect on male HCWs.

As with previous studies ${ }^{59}$ our nurses had the highest frequency of hand hygiene (69\%). This could be because most nurses are female or because of an emphasis on hand washing in their undergraduate training. However, with our nurses hand hygiene did not increase following intervention. Possibly few nurses were present at the lecture, hence, they only received written information concerning initial study results.

As observed by others, ${ }^{569}$ we found hand hygiene among doctors was low (11\%). However, intervention had its greatest effect on the doctors $(p=0.01)$. Although numbers are small, intervention had a positive effect on manipulative procedures, especially when using 5-fluorouracil.

Our study demonstrates that hospital policy is not being practised. Getting HCWs to clean their hands has been an ongoing struggle since Semmelweis. It has been suggested that patients should ask their healthcare professional to hand wash. ${ }^{10}$ Although controversial, this may help in the eradication of hospital acquired infection.

E Mensah, I E Murdoch, K Binstead, C Rotheram, W Franks Moorfields Eye Hospital NHS Foundation Trust, City Road, London ECIV 2PD, UK

Correspondence to: E Mensah, Moorfields Eye Hospital NHS Foundation Trust, City Road, London ECIV 2PD, UK; eveosh@aol.com

Local ethics approval was obtained for this study. doi: 10.1136/bjo.2005.072538 Accepted for publication 21 May 2005

Table 1 Effect of intervention on hand hygiene compliance

\begin{tabular}{|c|c|c|c|}
\hline & \multirow{2}{*}{$\begin{array}{l}\text { Hand hygiene before intervention } \\
\text { No (\%) }\end{array}$} & \multirow{2}{*}{$\begin{array}{l}\text { Hand hygiene after } \\
\text { intervention } \\
\text { No (\%) }\end{array}$} & \multirow[b]{2}{*}{ p Value } \\
\hline & & & \\
\hline Hand hygiene opportunities & 249 & 291 & \\
\hline Hand hygiene episodes before patient contact & $36(14)$ & $73(25)$ & 0.002 \\
\hline Hand hygiene episodes during patient contact & $8(3)$ & $8(3)$ & 1.00 \\
\hline Total hand hygiene episodes & $44(18)$ & $81(28)$ & 0.005 \\
\hline Hand hygiene episodes for procedures & $2 / 7(0 / 1$ for $5-\mathrm{FU})$ & $6 / 7(3 / 3$ for $5-\mathrm{FU})$ & 0.04 \\
\hline \multicolumn{4}{|l|}{ Sex of healthcare worker } \\
\hline Female & $32 / 107(30)^{*}$ & $62 / 115\left(54^{* *}\right.$ & $<0.001$ \\
\hline Male & $12 / 133(9)^{*}$ & $20 / 182(11)^{\star *}$ & 0.57 \\
\hline \multicolumn{4}{|l|}{ Profession of healthcare worker } \\
\hline Doctor & $21 / 191(11)$ & $44 / 220(20)$ & 0.01 \\
\hline Nurse & $18 / 26(69)$ & $25 / 43(58)$ & 0.36 \\
\hline Optometrist & $3 / 19(16)$ & $8 / 26(31)$ & 0.25 \\
\hline Other & $2 / 8(25)$ & $1 / 7(14)$ & 0.62 \\
\hline \multicolumn{4}{|c|}{$\begin{array}{l}\text { Only three out of nine examination bays were observed for } 1 \text { hour at a time, in random order, during morning (from } 09: 30 \text { to } 12: 30 \text { ) and afternoon (from } 14: 00 \\
\text { to } 17: 00 \text { ) clinics. } \\
\text { Data were analysed using } \chi^{2} \text { contingency tests. } \\
5 \text {-FU }=5 \text {-fluorouracil. } \\
{ }^{*} \mathrm{p}<0.001 ;{ }^{* *} \mathrm{p}<0.001 \text {. }\end{array}$} \\
\hline
\end{tabular}




\section{References}

1 Bauer TM, Ofner E, Just HM, et al. An epidemiological study assessing the relative importance of airborne and direct contact transmission of microorganisms in a medical intensive care unit. J Hosp Infect 1990; 15:301-9.

2 Bischoff WE, Reynolds TM, Sessler CN, et al. Handwashing compliance by health care workers. The impact of introducing an accessible, alcoholbased hand antiseptic. Arch Intern Med 2000; 160:1017-21.

3 Tiemersma EW, Bronzwaer SLAM, Lyytikainen O et al. Methicillin-resistant Staphylococcus aureus in Europe, 1999-2002. Emerg Infect Dis 2004; 10: 1627-34.

4 Pittet D, Simon A, Hugonnet S, et al. Hand hygiene among physicians: performance, beliefs, and perceptions. Ann Intern Med 2004:141:1-8.

5 Hugonnet S, Perneger TV, Pittet D. Alcohol-based handrub improves compliance with hand hygiene in intensive care units. Arch Intern Med 2002: 162:1037-43.

6 van de Mortel T, Bourke R, McLoughlin J, et al. Gender influences handwashing rates in the critical care unit. Am J Infect Control 2001;29:395-9.

7 Eagly AH. Sex differences and social behaviour: a social role interpretation. Hillsdale, NJ: Erlbaum, 1987

8 Johnson HD, Sholcosky D, Gabello K, et al. Sex differences in public restroom handwashing behaviour associated with visual behaviour prompts. Percept Mot Skills 2003;97(3 $\mathrm{Pt1}$ ):805-10.

9 Pittet D, Hugonnet S, Harbarth S, et al. Effectiveness of a hospital-wide programme to improve compliance with hand hygiene. Infection control programme. Lancet 2000;356:1307-12.

10 Jarvis WR. Handwashing - the Semmelweis lesson forgotten? Lancet 1994;344:1311-12.

\section{Successful treatment of Wegener's granulomatosis associated scleritis with rituximab}

Rituximab (Rituxan, Genentech, Inc, South San Francisco, CA, USA) is a new anti-CD20 $\mathrm{B}$ cell monoclonal antibody that has been used successfully to treat refractory cases of Wegener's granulomatosis (WG). ${ }^{1-3}$ There has been no published report of its effect in Wegener's associated eye disease. We describe the successful treatment of Wegener's associated scleritis with rituximab.

\section{Case report}

A 21 year old man with WG, proved on renal biopsy and by anti-neutrophil cytoplasm antibody (ANCA) positivity 6 years earlier, presented with bilateral, painful, red eyes. On examination his visual acuities were $6 / 4$ right eye and 6/5 left eye. Anterior segment examination showed subconjunctival haemorrhage, congested scleral vessels, scleral oedema, peripheral corneal infiltrates, and mild anterior chamber inflammation in each eye. Funduscopy revealed bilateral swollen optic discs with scattered retinal haemorrhages in the right eye. A diagnosis of scleritis was made. Oral prednisolone was increased from 5-40 mg daily and maintenance oral mycophenolate mofetil $2 \mathrm{~g}$ daily was continued. Topical prednisolone acetate $1 \%$ hourly was commenced to both eyes.

Over the next month the scleritis had not improved and his systemic vasculitis had become more active, causing arthralgia, haemoptysis, and new vasculitic skin lesions His white cell count (WCC) had risen to $13.9 \times 10^{9}$ compared to $9.6 \times 10^{9}$ the previous month. His ANCA had become positive by indirect immunofluorescence (titre of 1 in
25 ), and by proteinase 3 specific ELISA (titre 22 units, normal range $<10$ ). A new infiltrate was present in the lower lobe of his right lung on chest $x$ ray.

Owing to concern over the total cumulative dose of cyclophosphamide he had previously received $(>25 \mathrm{~g})$, he was given an intravenous infusion of rituximab $\mathrm{l} \mathrm{g}$. Intravenous cyclophosphamide $(12.5 \mathrm{mg} / \mathrm{kg}$, adjusted for renal function) was also given with the rituximab infusion. These infusions were repeated after 2 weeks.

This led to an immediate significant systemic improvement accompanied by reduction of WCC to $9.6 \times 10^{9}$ and ANCA became undetectable. The pulmonary infiltrate resolved. The scleritis also resolved promptly, evident from completely white eyes, resolution of active scleral vessels, corneal infiltrates, optic disc swelling, and subjective resolution of ocular pain. At 7 months after the infusion, the patient remained in remission. His systemic treatment was slowly reduced to prednisolone $15 \mathrm{mg}$ daily and mycophenolate mofetil $750 \mathrm{mg}$ twice daily.

\section{Comment}

Rituximab is a humanised monoclonal antibody against the CD20 antigen that is expressed on the cell surface during early pre-B cell development and persists through all stages of B cell differentiation. ${ }^{4}$ It results in rapid depletion of $\mathrm{CD} 20$ positive $\mathrm{B}$ lymphocytes from the circulating blood and is well tolerated. The precise role of B cells in the pathogenesis of WG remains elusive at present, but several possibilities exist. B cells can act as antigen presenting cells to T cells or provide additional co-stimulatory signals for them. Another possibility is that self reactive B cells, derived from unusual B cell subsets, may follow an alternative maturation process, including the continued expression of CD20 during antibody production.

There has been no report on its effect on WG associated scleritis. Our patient was given rituximab primarily for his generalised vasculitis, but his refractory scleritis also responded promptly. Although he also received cyclophosphamide at the same time, the dose and course were limited to avoid toxicity. Therefore, in this case the prompt improvement was attributed to rituximab, rather than cyclophosphamide.

This is the first case reporting rituximab as an effective treatment for refractory WG associated scleritis.

C M G Cheung, P I Murray Ophthalmology, Division of Immunity and Infection, University of Birmingham, Birmingham, UK

C O S Savage

Nephrology, Division of Immunity and Infection, University of Birmingham, Birmingham, UK

Correspondence to: Professor P I Murray, Academic Unit of Ophthalmology, Birmingham and Midland Eye Centre, City Hospital, Dudley Road, Birmingham B18 7QU, UK; p.i.murray@bham.ac.uk

doi: 10.1136/bjo.2005.075689

Accepted for publication 19 June 2005

\section{References}

1 Specks U, Fervenza FC, McDonald TJ, et al. Response of Wegener's granulomatosis to antiCD20 chimeric monoclonal antibody therapy. Arthritis Rheum 2001;44:2836-40.

2 Keogh KA, Wylan ME, Stone JH, et al. Induction of remission by $\mathrm{B}$ lymphocyte depletion in eleven patients with refractory antineutrophil cytoplasmic antibody-associated vasculitis. Arthritis Rheum 2005;52:262-8

3 Ferraro AJ, Day CJ, Drayson MT, et al. Effective therapeutic use of rituximab in refractory Wegener's granulomatosis. Nephrol Dia Transplant 2005;20:622-5.

4 Stashenko P, Nadler LM, Hardy R, et al. Characterization of a human $B$ lymphocytespecific antigen. J Immunol 1980;125:1678-85.

5 Chumley MJ, Dal Porto JM, Cambier JC. The unique $\mathrm{Ag}$ receptor signaling phenotype of B-1 cells is influenced by locale but induced by antigen. J Immunol 2002;169:1735-43.

\section{Retinopathy is not the only ocular symptom: myasthenia gravis in association with interferon therapy}

Interferons (IFNs) have antiviral and antimitogenic effects and are often used in the treatment of viral hepatitis or some neoplasms. However, they have various side effects including fever, nausea, depression, retinopathy, and autoimmune diseases. Although myasthenia gravis (MG) is rarely associated with IFN therapy, some cases developing MG after IFN or IFN/ribavirin combined therapy for chronic active hepatitis $\mathrm{C}$ have been reported. ${ }^{1-5}$ We report such a case by reviewing the clinical data.

\section{Case report}

A 69 year old man with chronic hepatitis C for 11 years had been treated with IFN- $\alpha$ monotherapy (IFN $6 \times 10^{6}$ IU three times a week after 2 weeks of daily injections). The first treatment started in April 2002. There was no complication noted in that treatment. After the therapy hepatitis $\mathrm{C}$ virus activity settled for a while, but during the observation his clinical data showed a rise in hepatitis $\mathrm{C}$ virus RNA and aminotransferases. He underwent IFN- $\alpha$ therapy conjugated with ribavirin $\left(\right.$ IFN $6 \times 10^{6}$ IU three times a week after 2 weeks of daily injections, ribavirin $800 \mathrm{mg}$ twice a day) again on 6 December 2002. During the course his condition was checked periodically, mainly in terms of retinopathy. He had finished 7 months of treatment without significant side effects.

Around December 2003 he began to notice fluctuating diplopia. Examination revealed his reduced right adduction, exotropia and left/ right hypertropia. Since his condition drifted and there was no significant disorder on magnetic resonance imaging, MG was suspected and edrophonium chloride was tested. With the medication, his diplopia prominently improved and MG was diagnosed; however, there was no elevation in his anti-acetylcholine receptor antibody titre or other auto-antibodies, and thymoma was not detected.

\section{Comment}

It is well known that IFN therapy induces autoimmunity. Thyroid auto-antibodies are the most frequent findings; autoimmune hepatitis, rheumatoid symptoms, induction of insulin dependent diabetes, etc, are also seen. In relation to this autoimmune effect, several cases concerning MG associated with IFN therapy have been reported. Some cases developed myasthenia newly or others exacerbated pre-existing symptoms. ${ }^{1-5}$ It is reported that cases with pre-existing $M G$ have a tendency to present more severe symptoms including myasthenic crisis. ${ }^{4}$ The pathogenesis is not completely understood 
because of the complex immunological effects of IFNs, including enhanced lymphocyte cytotoxicity, inhibition of $\mathrm{T}$ suppressor cell function, increased expression of major histocompatibility complex (MHC) class I antigens, production of proinflammatory cytokines, and differentiation of antigen presenting cell activation of $\mathrm{T}$ helper lymphocytes by autoantigens. Some or all of them might contribute to the development of autoimmune disease. ${ }^{6}$

In this case the patient had no sign of MG or other autoimmune disease before the IFN treatment. His symptom is limited only to extraocular muscles: the condition is relatively mild. That is consistent with the previous report referring to the relation between the severity and the presence of a history of autoimmune disease; but the fact that anti-acetylcholine receptor antibody titre was not elevated is contradictory. ${ }^{4}$ We could not establish the causality.

These days many patients with chronic active hepatitis $C$ virus receive IFN or IFN/ribavirin combined therapy. We usually examine these patients only in terms of retinopathy. Although this case could be a coincidental sporadic autoimmune disorder, we should take MG into consideration. We should recognise the risk of development or worsening of MG and be careful in managing patients undergoing therapy, especially when they already have MG or compatible symptoms. It can be a serious complication although it is very rare.

A Oishi, K Miyamoto, S Kashii, N Yoshimura Department of Ophthalmology and Visual Sciences, Kyoto University Graduate School of Medicine, Kyoto, Japan

Correspondence to: Dr Akio Oishi, Department of Ophthalmology and Visual Sciences, Kyoto University Graduate School of Medicine, Kyoto, Japan; aquio@ kuhp.kyoto-u.ac.jp

doi: 10.1136/bjo.2005.077537

Accepted for publication 1 July 2005

\section{References}

1 Gurtubay IG, Morales G, Arechaga O, et al. Development of myasthenia gravis after interferon alpha therapy. Electromyogr Clin Neurophysiol 1999:39:75-8.

2 Borgia G, Reynaud L, Gentile I, et al. Myasthenia gravis during low-dose IFN-alpha therapy for chronic hepatitis C. J Interferon Cytokine Res 2001;21:469-70.

3 Weegink CJ, Chamuleau RA, Reesink HW, et al. Development of myasthenia gravis during treatment of chronic hepatitis $C$ with interferonalpha and ribavirin. J Gastroenterol 2001;36:723-4.

4 Konishi T. A case of myasthenia gravis which developed myasthenic crisis after alpha-interferon therapy for chronic hepatitis C. Rinsho Shinkeigaku 1996;36:980-5.

5 Harada H, Tamaoka A, Kohno Y, et al. Exacerbation of myasthenia gravis in a patient after interferon $\beta$ treatment for chronic active hepatitis C. J Neurol Sci 1999;165:182-3.

6 Dumoulin FL, Leifeld L, Sauerbruch T, et al. Autoimmunity induced by interferon- $\alpha$ therapy for chronic viral hepatitis. Biomed Pharmacother 1999:53:242-54.

\section{Tobacco-alcohol amblyopia: a maculopathy?}

Tobacco-alcohol amblyopia or toxic-nutritional optic neuropathy is a condition characterised by papillomacular bundle damage, central or caecocentral scotoma, and reduction of colour vision in a patient who abuses
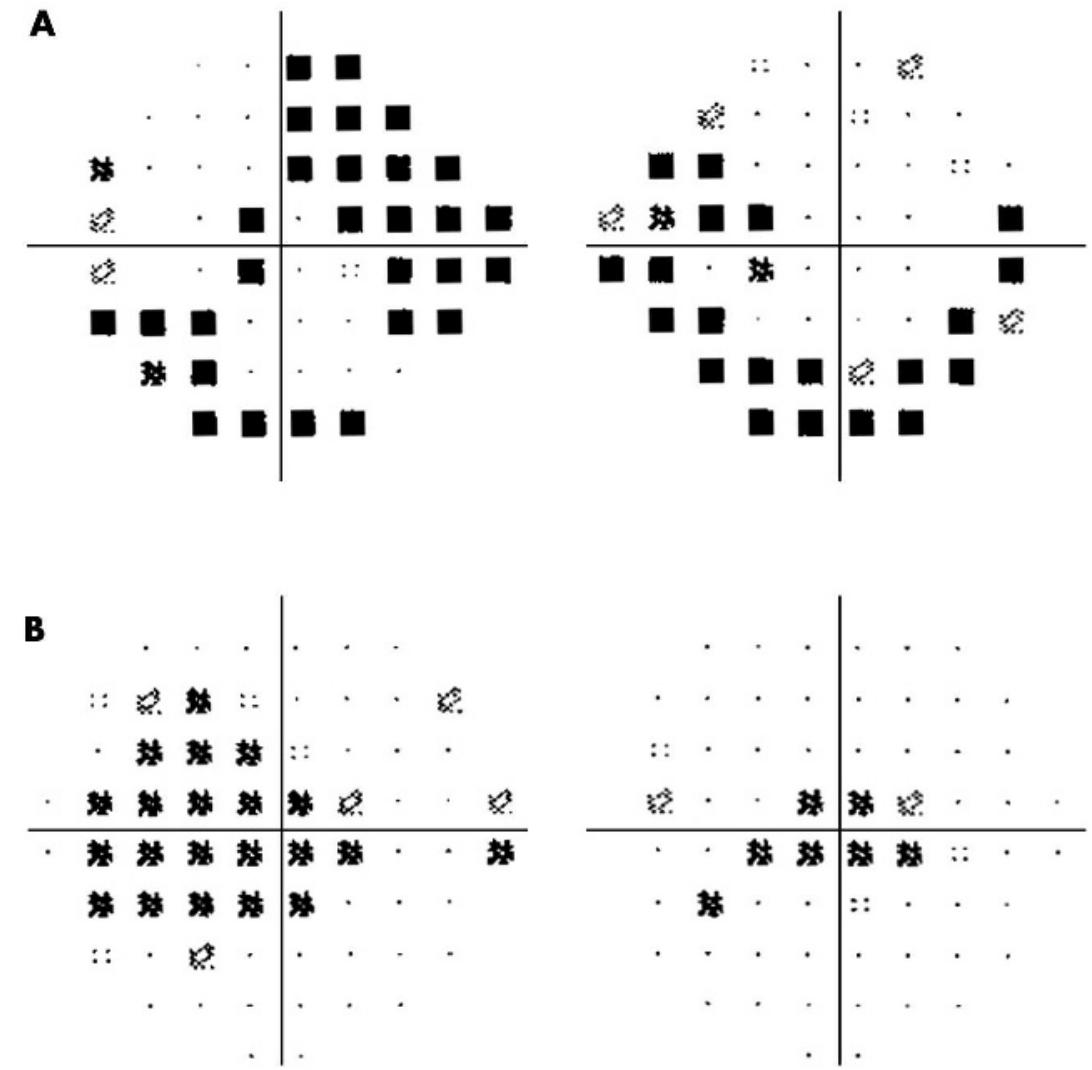

Figure 1 Static perimetry using (A) the 24-2 program shows an inferior arcuate defect in the right eye; a superonasal and an inferior defect in the left eye. (B) The 10-2 program shows bilateral central defects.

tobacco and alcohol. ${ }^{12}$ There is consensus that nutritional deficiency has an important role as well. ${ }^{34}$ The appearance of the optic nerve is usually normal, but peripapillary dilated vessels and haemorrhages have been described. ${ }^{5}$ Testing with static perimetry often reveals central scotomas. Although this syndrome has been classified as optic neuropathy, the primary lesion has not actually been localised to the optic nerve and may possibly originate in the retina, chiasm, or even the optic tracts. We report two cases of tobacco-alcohol amblyopia and their electrophysiological findings after testing with multifocal electroretinography (MERG).

\section{Case reports}

Case 1

A 47 year old woman presented with a gradual decrease in vision over 4 months. Her medical history showed that she has been in excellent health. She smoked one pack of cigarettes per week and had two to three beers daily. She denied any use of any medications in the past few months. She and her husband have been on a diet which contained fewer vegetables than their normal intake, for 4 months. Family history was unremarkable.

Visual acuity was 20/50 right eye and 20/100 left eye. Colour vision using the pseudoisochromatic plates was four of eight in right eye and two of eight in left eye. Intraocular pressure was $12 \mathrm{~mm} \mathrm{Hg}$ right eye and $15 \mathrm{mmHg}$ left eye. She had normal anterior segment in both eyes. Her pupils were sluggish to direct stimulation of light with no afferent defect. Ocular motility was normal. Funduscopy showed anomalous optic nerves with no pallor, and normal maculas. Testing with 24-2 static perimetry revealed an inferior and nasal defect in the right eye; superonasal, inferior, and central defect in the left eye (fig 1A). Humphrey 10-2 static perimetry showed bilateral caecocentral scotomas (fig 1B). Magnetic resonance imaging (MRI) of the brain and orbit with and without contrast was normal. Serology tests for Lyme and antinuclear antibodies (ANA) were negative. Complete blood count, serum vitamin B12, and folate were within normal limits. MERG testing showed severe reduction in amplitude mostly centrally in both eyes (fig 2).

\section{Case 2}

A 55 year old woman presented with progressive decrease in vision of both eyes over 1 month. She had a history of multiple intracranial aneurysms that were clipped 15 years earlier. She was not using any medications. She smoked one pack of cigarette a day for 25 years and has five to eight drinks per week. Family history was positive for glaucoma in her mother. Visual acuity was counting fingers at $\mathrm{l}$ foot right eye and at 2 feet left eye. She could not identify any of the pseudoisochromatic colour plates in both eyes. She had normal anterior segment in both eyes. Pupillary reactions were sluggish to light stimulation with no afferent defect. Funduscopy showed mildly swollen optic nerves in both eyes (fig 3). Kinetic perimetry 

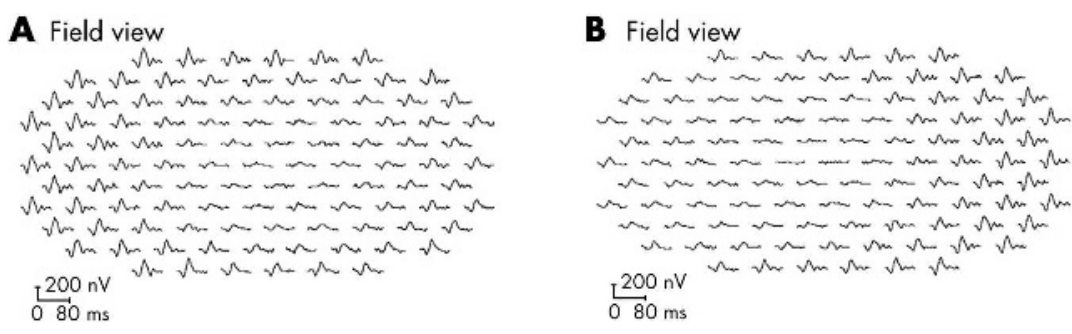

Figure 2 Trace arrays of multifocal ERG showing decreased amplitudes in both the right eye (A) and the left eye (B) with almost isoelectric responses centrally and improvement towards the periphery.
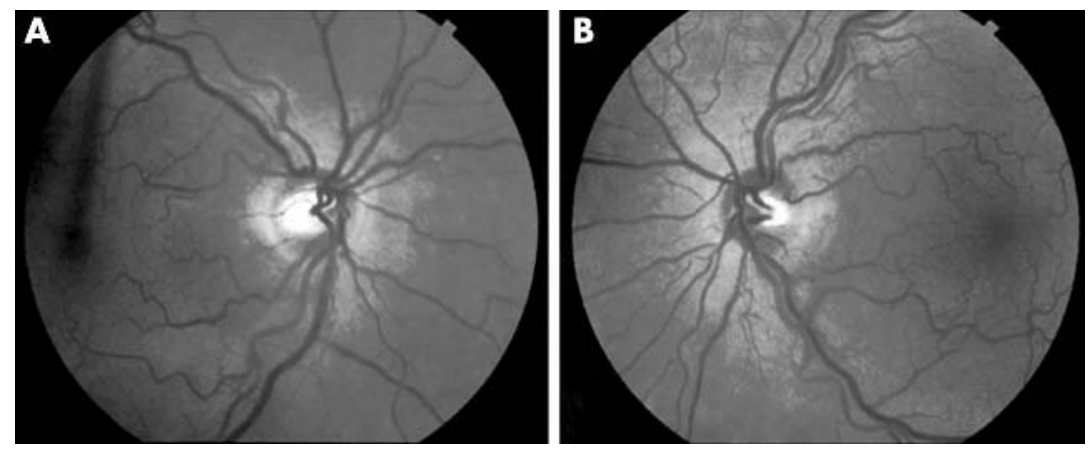

Figure 3 Fundus photographs showing swollen discs with some telangiectatic vessels in both eyes. (A) Right eye, (B) left eye.
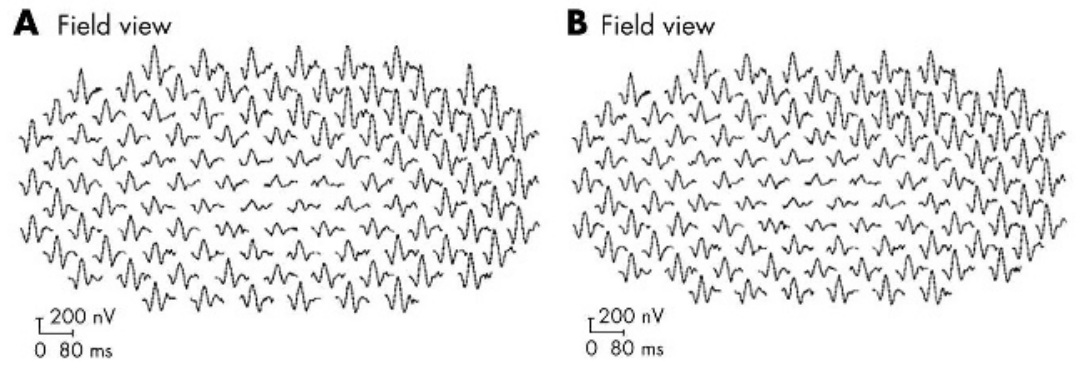

Figure 4 Trace arrays of patient 2 showing reduced amplitude in the central region of both eyes.

showed bilateral central scotomas. A CT scan (with and without contrast agent) of the brain and orbit was normal. Complete blood count, serum vitamin B12, and folate were within normal limits. Genetic testing of mitochondrial DNA for Leber's hereditary optic neuropathy showed that the patient has the LHON $3460 \mathrm{G}$ mutation. Multifocal ERG was performed and showed decreased amplitudes centrally with normal latency (fig 4).

\section{Comment}

We describe two cases of "tobacco-alcohol amblyopia in patients who had a history of high alcohol intake (cases 1 and 2) and shortly after dietary alteration (case 1). In both cases, MERG testing showed decreased amplitudes in the central region, suggesting retinal dysfunction in the macula. The condition of the patient in case 2 may have been precipitated by a metabolic injury (tobacco, alcohol) to genetically "compromised" mitochondria. This shows the clinical overlap in conditions of inherited mitochondrial dysfunction and acquired ones such as tobacco-alcohol amblyopia.
The clinical findings seen in tobaccoalcohol amblyopia can occur in any disease of anterior visual pathway from the retina to the optic tract and there is there is little evidence to suggest that the locus of pathology is restricted to the optic nerve. Hhistopathological studies on animal models of nutritional amblyopia showed lesions in the retina, optic nerve and tract, ${ }^{8}$ and the maculopapillary bundle. ${ }^{9}$ Electrophysiological abnormalities in animal models of tobaccoalcohol amblyopia showed reduced amplitudes with normal latencies using visual evoked potentials, ${ }^{10}{ }^{11}$ and increased a-wave and b-wave implicit times and decreased bwave amplitudes using full field electroretinograms. ${ }^{12}$ the outer retina (photoreceptor and bipolar cell layer) with only minimal contribution from the inner retina and optic nerve (ganglion cells and nerve fibre layer)..$^{13}$ Therefore, the severe reduction in amplitude in our patients suggests that the outer retina, particularly in the macula, is involved in this condition.
MERG signals are believed to arise from
R Behbehani, R C Sergott, P J Savino Neuroophthalmology Service, Wills Eye Hospital, Thomas Jefferson University, Philadelphia, PA, USA

Correspondence to: Raed Behbehani, MD, PO Box 1262, 130013 Safat, Kuwait; r_behbehani@hotmail.com

doi: 10.1136/bjo.2005.079137 Accepted for publication 18 July 2005

The authors have no proprietary interest in any of the instruments used or any other aspect of this study.

\section{References}

1 Traquair HM. Toxic amblyopia, including retrobulbar neuritis. Trans Ophthalmol Soc UK 1930;50:351.

2 Golnik KC, Schaible ER. Folate-responsive optic neuropathy. J Neuro-ophthalmol 1994;14:163.

3 Victor M. Tobacco-alcohol amblyopia. A critique of current concepts of this disorder, with special reference to the role of nutritional deficiency in its causation. Arch Ophthalmol 1963:70:313.

4 Victor M, Mancall EL, Dreyfus PM. Deficiency amblyopia in the alcoholic patient: a clinicopathologic study. Arch Ophthalmol 1960;64:1.

5 Carroll FD. Nutritional amblyopia. Arch Ophthalmol 1966;76:406.

6 Frisén L. Fundus changes in acute nutritional amblyopia. Arch Ophthalmol 1983;101:577.

7 Cullom ME, Hayes KL, Miller NR, et al. Leber's hereditary optic neuropathy masquerading as tobacco-alcohol amblyopia. Arch Ophthalmol 1993; 111:1482.

8 Rodger FC. Degenerative changes in the rat visual pathway when thiamine and riboflavin deficiencies are combined. Br J Ophthalmol 1954;38:154.

9 Smiddy WE, Green WR. Nutritional amblyopia. A histopathologic study with retrospective clinical correlation. Graefes Arch Clin Exp Ophthalmol 1987;225:321-4.

10 Ikeda H, Tremain KE, Sanders MD. Neurophysiological investigation in optic nerve disease: Combined assessment of the visual evoked response and electroretinogram. Br J Ophthalmol 1978;62:227.

11 Kupersmith MJ, Weiss PA, Carr RE. The visual evoked potential in tobacco-alcohol amblyopia and nutritional amblyopia. Am J Ophthalmol 1983;95:307.

12 Pawlosky RJ, Bacher J, Salem N Jr. Ethanol consumption alters electroretinograms and depletes neural tissues of docosahexaenoic acid in rhesus monkeys: nutritional consequences of a low n-3 fatty acid diet. Alcohol Clin Exp Res 2001;25: 1758-65.

13 Hood DC, Odel JG, Chen CS, et al. The multifocal electroretinogram. J Neuro-ophthalmol 2003;23:225-35.

\section{MAILBOX}

\section{TTT: local light absorption and heat convection versus heat conduction}

Miura and co-authors have contributed valuable experimental data on transpupillary thermotherapy (TTT) for choroidal neovascularisation (CNV) in a rat model. ${ }^{1}$ In their scholarly discussion section, they speculate that the variability in power settings they encountered in heating experimental CNV may be due to a "variation of heat conduction in experimental CNV."1 There are more probable explanations for that variability. As reported previously in the authors' reference 7: "light absorption in pigment clumps from 
prior focal photocoagulation can cause local hot spots in large TTT treatment fields." Additionally, local choroidal blood flow ${ }^{2}$ may have been altered by vascular remodelling that occurred in the 14 days between the intense focal laser photocoagulation that the authors used to produce CNV and their subsequent liposomal monitored TTT at the site.

Chorioretinal temperature rise from a lengthy 60 seconds TTT exposure is affected: (1) by pigmentation at the treatment site, which determines how effectively laser radiant energy is converted locally into thermal energy, and (2) to a lesser extent by choroidal blood flow, ${ }^{3}$ which transfers thermal energy by heat convection away from the exposure site. It is unlikely that local heat conduction is altered significantly by the initial photocoagulation or subsequent tissue remodelling because heat conduction in most normal biological tissues is essentially the same as that of water. ${ }^{4-6}$

M A Mainster

Department of Ophthalmology, University of Kansas Medical School, 3901 Rainbow Boulevard, Kansas City, KS 66160-7379, USA

D H Silney

Laser/Optical Radiation Program, US Army Environmental Hygiene Agency, Aberdeen Proving Ground, MD 21010, USA

Correspondence to: Professor Martin A Mainster, Department of Ophthalmology, M53009, University of Kansas Medical School, 3901 Rainbow Boulevard Kansas City, KS 66160-7379, USA mmainster@kumc.edu

doi: $10.1136 /$ bjo.2005.082453

Accepted for publication 6 September 2005

\section{References}

1 Miura S, Nishiwaki H, leki $Y$, et al. Chorioretinal temperature monitoring during transpupillary thermotherapy for choroidal neovascularisation. Br J Ophthalmol 2005;89:475-9.

2 Mainster MA, Reichel E. Transpupillary thermotherapy for age- related macular degeneration: long-pulse photocoagulation, apoptosis, and heat shock proteins. Ophthalmic Surg Lasers 2000;31:359-73.

3 Welch AJ, Wissler EH, Priebe LA. Significance of blood flow in calculations of temperature in laser irradiated tissue. IEEE Trans Biomed Eng 1980:27:164-6.

4 Mainster MA, White TJ, Tips JH, et al. Retinaltemperature increases produced by intense light sources. J Opt Soc Am 1970;60:264-70.

5 Welch AJ, van Gemert MJC. Optical-thermal response of laser-irradiated tissue. New York: Plenum Press, 1995

6 Mainster MA. Decreasing retinal photocoagulation damage: principles and techniques. Sem Ophthalmol 1999;14:200-9.

\section{NOTICES}

\section{World Ophthalmology Congress 2006 - Brazil}

The World Ophthalmology Congress (which is replacing the International Congress of Ophthalmology) is meeting in February 2006 in Brazil.

For further information on the congress and committees, scientific program and coordinators of different areas are available at the congress website www.ophthalmology2006. com.br

\section{Vision 2020}

The latest issue of Community Eye Health (No 54) assesses the progress of Vision 2020 at the district level. For further information please contact: Journal of Community Eye Health, International Resource Centre, International Centre for Eye Health, Department of Infectious and Tropical Diseases, London School of Hygiene and Tropical Medicine, Keppel Street, London WClE 7HT, UK (tel: +44 (0)20 7612 7964; email: Anita.Shah@1shtm.ac.uk; online edition: www.jceh.co.uk). Annual subscription (4 issues) UK $£ 28 / U S \$ 45$. Free to developing country applicants.

\section{$19^{\text {th }}$ International Society for Geographical \& Epidemiological Ophthalmology Congress}

The $19^{\text {th }}$ ISGEO congress will be held in Sao Paulo, Brazil on 18-19 February, 2006, just prior to the ICO. Abstract submission and registration forms can be obtained by emailing Dr Paul Courtright (pcourtright@kcco. net, url: ) or by accessing the ISGEO website at www.kcco.net/isgeo.

\section{Medical Contact Lens \& Ocular surface Association Winter Symposium}

The MCLOSA Winter Symposium will be held at the Western Eye Hospital, Marylebone Road, London NWl on Friday 18 November 2005. For further information please visit the MCLOSA website at www.mclosa.org.uk/ annualmtg.html.

\section{CORRECTIONS}

doi: 10.1136/bjo.2005.75895corr l

In the letter titled, Patient satisfaction with anaesthesia comparing sun-Tenon's block and topical anaesthesia $(B r \quad J$ Ophthalmol 2005;89:1228) the second author was omitted. The second author for this letter was R W D Bell, Sunderland Eye Infirmary, Queen Alexandra Road, Sunderland SR2 9HP, UK. The author apologises for this omission.

doi: 10.1136/bjo.2004.5894lcorr l

In the paper titled, En-face optical coherence tomography (OCT): A new method to analyse structural changes of the optic nerve head in rat glaucoma ( $B r J$ Ophthalmol 2005;89: 1210-6) one of the author's name has been spelt incorrectly. The author Podolean AG, should be spelt Podoleanu AG. The journal apologises for this error. 